NUMBER THEORY WEEK 2017

BANACH CENTER PUBLICATIONS, VOLUME 118

INSTITUTE OF MATHEMATICS

POLISH ACADEMY OF SCIENCES

WARSZAWA 2019

\title{
EVEN ASCENDING POWERS
}

\author{
JÖRG BRÜDERN \\ Mathematisches Institut, Universität Göttingen \\ Bunsenstrasse 3-5, D-37073 Göttingen \\ E-mail: bruedern@uni-math.gwdg.de
}

To Jurek, on the occasion of his sixtieth birthday

Abstract. Almost all natural numbers not divisible by eight are the sum of a square, a fourth, a sixth and an eighth power. This is established in quantitative form.

1. Introduction. In 1949 Klaus Roth introduced an aesthetically attractive variant of Waring's problem. He discussed representations of natural numbers as sums of ascending powers. With $s$ and $n$ natural numbers, consider the family of diophantine equations

$$
\sum_{j=1}^{s} x_{j}^{j+1}=n .
$$

Let $H$ be the smallest number $s$ such that for all sufficiently large $n$ there is a solution to (1.1) in natural numbers $x_{j}$, and let $H^{+}$be the smallest $s$ such that this is so for almost al $]^{1} n$. Roth [10] first showed that $H^{+}=3$ and then [11] added the bound $H \leq 50$. This last estimate spawned much work over decades, currently culminating in the inequality $H \leq 14$ of Ford [5]. It is conjectured that $H=3$, but this seems well beyond the scope of existing technology.

In this paper we consider Roth's problem with even powers only. Let $H_{\mathrm{e}}$ be the smallest number $s$ such that, for all large $n$, the diophantine equations

$$
\sum_{j=1}^{s} x_{j}^{2 j}=n
$$

2010 Mathematics Subject Classification: Primary 11P05; Secondary 11P55.

Key words and phrases: Waring's problem, Hardy-Littlewood method, pruning.

The paper is in final form and no version of it will be published elsewhere.

${ }^{1} \mathrm{~A}$ certain statement concerning natural numbers holds for almost all $n$ if the proportion of those $n \leq N$ where the statement is true, tends to 1 as $N \rightarrow \infty$. 
have solutions in natural numbers $x_{j}$. Let $H_{\mathrm{e}}^{+}$be the smallest $s$ where the equations 1.2 have such solutions for almost all $n$. From the inequality

$$
\#\left\{(x, y, z) \in \mathbb{N}^{3}: x^{2}+y^{4}+z^{6} \leq N\right\} \leq N^{11 / 12}
$$

we infer that no more than $N^{11 / 12}$ of the natural numbers $n$ not exceeding $N$ can be written as $n=x^{2}+y^{4}+z^{6}$. Next, consider the equation

$$
x_{1}^{2}+x_{2}^{4}+x_{3}^{6}+x_{4}^{8}=n .
$$

Reduction modulo 16 shows that whenever $n \equiv 8 \bmod 16$ then there is no solution in integers $x_{j}$. In particular, there is a positive proportion of natural numbers $n$ that have no representation of the form 1.3 . This implies that $H_{\mathrm{e}}^{+} \geq 5$. However, an analysis of local solubility of 1.3 reveals that the only obstruction occurs in the 2-adic integers. Indeed, among the numbers that are not divisible by 8 , almost all are the sum of a square, a biquadrate, a sixth power and an eighth power of natural numbers. This is a consequence of our main result concerning the number $r(n)$ of solutions of (1.3) in natural numbers $x_{j}$.

THEOREM. There is a positive number $c$ with the property that whenever $N$ is sufficiently large, then

$$
r(n) \geq c n^{1 / 24}(\log \log 9 n)^{-1 / c}
$$

holds for all but at most $N^{91 / 93}$ exceptions among the numbers $n \not \equiv 0 \bmod 8$ with $n \leq N$.

The remarks preceding the theorem show that no such result can be expected to hold for the equation $x^{2}+y^{4}+z^{6}=n$. Further, we note that $H_{\mathrm{e}}^{+}=5$. This follows from the theorem by taking $x_{5}=0$ when $n \not \equiv 0 \bmod 8$, and $x_{5}=1$ when $8 \mid n$, in the equation

$$
x_{1}^{2}+x_{2}^{4}+x_{3}^{6}+x_{4}^{8}+x_{5}^{10}=n .
$$

A formal application of the Hardy-Littlewood method suggests an asymptotic formula for $r(n)$ of the shape

$$
r(n) \sim \frac{\Gamma\left(\frac{3}{2}\right) \Gamma\left(\frac{5}{4}\right) \Gamma\left(\frac{7}{6}\right) \Gamma\left(\frac{9}{8}\right)}{\Gamma\left(\frac{25}{24}\right)} \mathfrak{S}(n) n^{1 / 24}
$$

wherein

$$
\mathfrak{S}(n)=\sum_{q=1}^{\infty} \sum_{\substack{a=1 \\(a, q)=1}}^{q} \sum_{\substack{x_{j}=1 \\ 1 \leq j \leq 4}}^{q} e\left(\frac{a\left(x_{1}^{2}+x_{2}^{4}+x_{3}^{6}+x_{4}^{8}-n\right)}{q}\right)
$$

is the singular series associated with the equation $\sqrt{1.3}$. It turns out that $\mathfrak{S}(n)$ converges absolutely, and that $\mathfrak{S}(n)=0$ for $n \equiv 8 \bmod 16$, but there is a constant $D>1$ with $(\log \log 9 n)^{-D} \ll \mathfrak{S}(n) \ll(\log \log 9 n)^{D}$ for all $n \not \equiv 0 \bmod 8$. In particular, the lower bound for $r(n)$ recorded in the theorem is of the expected order of magnitude.

Our estimate for the number of exceptional $n$ in the theorem is probably far from the truth. The proposed asymptotic formula, if true, would show the number of exceptions be finite. At this time, a mean square approach via the circle method to this kind of questions is limited by square root cancellation in the minor arc estimate. Thus, at best, 
one could hope for an estimate like $O\left(N^{23 / 24}\right)$ for the size of the exceptional set. In terms of the exponent of $N$, the bound supplied in our theorem is therefore slightly better than half way between what square root cancellation would give, and a merely qualitative almost all estimate.

The proof of the theorem proceeds by applying the circle method to a modified count of the solutions to 1.3 , with the sixth power restricted to smooth numbers. For the approach to succeed, we have to introduce unconventional elements to the treatment of both the minor and the major arcs. These novel features should impact the theory well beyond this communication, and we therefore describe them here in general terms but for a detailed account the reader is referred to later sections of this paper.

The minor arc analysis is performed in mean square over $n$. It is here where the square root cancellation barrier is relevant. The method rests on an elaborate pruning process. The driving force behind pruning is an amplification method. Hitherto, one would start with an application of Weyl's inequality or some variant thereof, outside a rather large set of major arcs. On these major arcs, the Weyl sum in question is well approximable by certain complete Weyl sums, and this allows one to compute certain low moments over major arcs much more precisely than would be possible for moments over $\mathbb{R} / \mathbb{Z}$. For an example and more comment on this matter, see 2.15 below, and the remarks that follow that estimate. Since weaker estimates of Weyl's type will now suffice to produce acceptable bounds, we end up with slimmer major arcs. This strategy, often in iterated form, was a fruitful one in numerous applications.

Classical pruning, however, seems to be deemed to failure when applied to (1.3). Instead, we replace the role of Weyl's inequality partly with a different procedure. We begin by postulating upper bounds on sixth and eighth power Weyl sums that are far stronger than currently available Weyl bounds. Based on this assumption, routine pruning is possible along the lines indicated in the preceding paragraph. We then explore the opposite situation where the Weyl sums are large (but not very large) through an argument of Chebyshev's type, leading to improved moment estimates. As soon as these moments are better controlled than what is known for these moments over $\mathbb{R} / \mathbb{Z}$, then one has another amplifier at hand. The amplified moment can be recycled in a recursive process that again leads to a pruning device. In favourable circumstances, this machinery provides a workable tool in situations outside the scope of the traditional approach. The work in $\S 3$ appears to be the first successful implementation of such ideas. The new method is applied twice, in an initial pruning with a smooth sixth power Weyl sum, and on certain intermediate arcs with a classical eighth power Weyl sum. There are many other possible uses for this new device, as we intend to demonstrate in a forthcoming article.

On the major arcs, we proceed in two steps. We work in mean square over $n$ while approximating all standard Weyl sums by complete ones. This is routine, and it separates a truncated kind of singular series for a square, a biquadrate and an eighth power from a similarly modified singular integral. We will then have to deal with the smooth sixth power. Here we are forced to work for individual $n$, because of our poor understanding of the distribution of smooth numbers in arithmetic progressions. It seems that at the current state of affairs with the latter, any attempt to work an average over $n$ will result in 
woefully weak estimates for the exceptional set. We therefore indeed consider the already modified form of the major arc contribution for individual $n$. After a series of somewhat unusual transformations, we will be able to bring in an estimate of Bombieri-Vinogradov type for smooth numbers. Fortunately, this can be imported from the work of Wolke [16], for example. We will then be able to extract a leading term that factorises into singular series and singular integral in the expected way.

The idea of using results on the distribution of smooth numbers in arithmetic progressions on average is not entirely new. This writer has applied a related technique within his work on sums of four cubes [3], almost thirty years ago. On that occasion, we relied on the principles behind a character sum approach to versions of the Barban-Davenport-Halberstam theorem for general sequences, rather than the BarbanDavenport-Halberstam theorem itself. In principle, this is a superior approach because much inflated major arcs are acceptable to variance methods, but for the problem at hand, the somewhat complicated machinery seems to be at odds with the inhomogeneity of the diophantine equation. We therefore propose a radically different implementation that brings in the average distribution over arithmetic progressions directly. This simplifies matters considerably, and one can choose to work with variances or estimates of Bombieri-Vinogradov type. We express our hope that the renovated design helps to popularize the method among workers on Waring's problem and its variants.

We have not said anything about $H_{\mathrm{e}}$ yet. The Freiman-Scourfield theorem [7, 12] shows that $H_{\mathrm{e}}$ is finite. A decent estimate for $H_{\mathrm{e}}$ can be obtained by combining the methods of this paper with those of Ford [5], but limitations of space do not permit to include an account of this here.

2. The arsenal. In this section we collect together standard estimates for Weyl sums, and fix some notation. Further, we present some applications of the pruning lemma. The material included here is for frequent use in the sequel, while more refined weaponry will be developed in due course.

Our primary parameter is $N$, a large real number. We apply the familiar convention concerning the letter $\varepsilon$ : whenever the letter $\varepsilon$ occurs in a statement, it is asserted that the statement is true for any fixed positive value assigned to $\varepsilon$. Should the statement involve a Landau or Vinogradov symbol, then the implicit constant will depend on $\varepsilon$. Note that with this convention we are allowed to conclude from $A \ll N^{\varepsilon}, B \ll N^{\varepsilon}$ that $A B \ll N^{\varepsilon}$, for example. In some parts of this work we apply a similar convention concerning the letter $C$ : a statement involving $C$ is valid provided that $C$ is sufficiently large. We will use this only finitely often, and therefore $C$ can be chosen to be the same number throughout.

Let $k$ be a natural number, $k \geq 2$. Let $P_{k}=N^{1 / k}$ and define the Weyl sum

$$
f_{k}(\alpha)=\sum_{x \leq P_{k}} e\left(\alpha x^{k}\right)
$$

Its adelic approximation involves

$$
S_{k}(q, a)=\sum_{x=1}^{q} e\left(\frac{a x^{k}}{q}\right), \quad v_{k}(\beta)=\frac{1}{k} \sum_{m \leq N} m^{1 / k-1} e(\beta m),
$$


and whenever $a \in \mathbb{Z}, q \in \mathbb{N}$ and $\beta \in \mathbb{R}$ one has

$$
f_{k}\left(\frac{a}{q}+\beta\right)=q^{-1} S_{k}(q, a) v_{k}(\beta)+O\left(q^{\varepsilon}(q+N q|\beta|)^{1 / 2}\right),
$$

see Vaughan [14, Theorem 4.1]. If $|\beta| \leq \frac{1}{2}$ then (Vaughan [14, Lemma 2.8])

$$
v_{k}(\beta) \ll P_{k}(1+N|\beta|)^{-1 / k} \text {. }
$$

The simple upper bound ([14, Theorem 4.2])

$$
q^{-1} S_{k}(q, a) \ll q^{-1 / k} \quad((a, q)=1)
$$

is often not good enough for us. Let $\kappa_{k}$ be the multiplicative function that, for primes $p$ and $u \geq 0,2 \leq v \leq k$, is defined by

$$
\kappa_{k}\left(p^{1+u k}\right)=k p^{-u-1 / 2}, \quad \kappa_{k}\left(p^{v+u k}\right)=p^{-u-1} .
$$

Then, by Lemmata 4.3 and 4.4 of Vaughan [14, one has

$$
q^{-1} S_{k}(q, a) \ll \kappa_{k}(q) \quad((a, q)=1),
$$

and directly from the definition we see that

$$
\sum_{q \leq Q} \kappa_{k}(q)^{2} \leq \prod_{p \leq Q}\left(1+\frac{k^{2}}{p}+O\left(\frac{1}{p^{2}}\right)\right) \ll Q^{\varepsilon} .
$$

Besides the familiar lemma of Hua we only require the elementary mean values

$$
\int_{0}^{1}\left|f_{2}(\alpha) f_{4}(\alpha)^{2}\right|^{2} \mathrm{~d} \alpha \ll N^{1+\varepsilon}, \quad \int_{0}^{1}\left|f_{2}(\alpha) f_{4}(\alpha) f_{8}(\alpha)^{2}\right|^{2} \mathrm{~d} \alpha \ll N^{1+\varepsilon} .
$$

that are special cases of [1, Lemma 1].

We now turn to moment estimates for smooth Weyl sums of degree 6 . Let $\mathcal{A}(P, R)$ denote the set of natural numbers not exceeding $P$ with all their prime factors below $R$. Then put

$$
g(\alpha, P, R)=\sum_{x \in \mathcal{A}(P, R)} e\left(\alpha x^{6}\right) .
$$

Write $\delta(3)=\frac{1}{11}, \delta(4)=\frac{1}{3}$ and $\delta(5)=0.7247$. Then, for any $\varepsilon>0$ there is a number $\eta>0$ with the property that the estimates

$$
\int_{0}^{1}\left|g\left(\alpha, P, P^{\eta}\right)\right|^{2 s} \mathrm{~d} \alpha \ll P^{s+\delta(s)+\varepsilon}
$$

hold for $s=3,4$ and 5. This follows from Vaughan [13] for $s=3$ and 4, and from the Appendix in Vaughan and Wooley [15] for $s=5$. Note here that $\eta$ depends on $\varepsilon$. However, a consideration of the underlying diophantine equations shows that if (2.9) holds for a particular value of $\eta$, then 2.9 is also valid for all smaller values of $\eta$. When applying our convention concerning the letter $\varepsilon$ we will call upon 2.9 only finitely often, so a fixed positive value of $\eta$, sufficiently small in terms of $\varepsilon$, will do. Having chosen this, we put

$$
g_{6}(\alpha)=g\left(\alpha, \frac{1}{2} P_{6}, P_{6}^{\eta}\right)
$$


Next, we introduce a Farey dissection of the unit interval $\mathfrak{U}=\left(N^{-1 / 2}, 1+N^{-1 / 2}\right]$. By Dirichlet's theorem, $\mathfrak{U}$ is contained in the union of intervals $\left\{\alpha:|q \alpha-a| \leq N^{-1 / 2}\right\}$ with

$$
1 \leq a \leq q \leq N^{1 / 2}, \quad(a, q)=1
$$

while it is easily seen that the shortened intervals $\left\{\alpha:|q \alpha-a| \leq \frac{1}{2} N^{-1 / 2}\right\}$ are disjoint when $a, q$ range over 2.11. Hence there are half-open intervals $\mathfrak{I}(q, a)=\left(\alpha_{q, a}^{\prime}, \alpha_{q, a}^{\prime \prime}\right]$ with

$$
\left\{\alpha:|q \alpha-a| \leq \frac{1}{2} N^{-1 / 2}\right\} \subset \Im(q, a) \subset\left\{\alpha:|q \alpha-a| \leq N^{-1 / 2}\right\}
$$

and the property that $\mathfrak{U}$ is their disjoint union as $a, q$ run over 2.11 . The exact location of $\alpha_{q, a}^{\prime}, \alpha_{q, a}^{\prime \prime}$ is of no relevance.

Let $1 \leq Q \leq N^{1 / 2}$, and define the major arcs $\mathfrak{M}(Q)$ as the union of the intervals

$$
\{\alpha \in \mathfrak{I}(q, a):|q \alpha-a| \leq Q / N\}
$$

with $1 \leq a \leq q \leq Q,(a, q)=1$. Note that for $Q \leq \frac{1}{2} N^{1 / 2}$ the intervals 2.12 are simply given by $|q \alpha-a| \leq Q / N$.

The standard pruning device is [2, Lemma 1]. The following reformulation is readier to use within this paper.

Lemma 1. Notation as above. Let $u_{l}$ be non-negative real numbers, and put

$$
U(\alpha)=\sum_{|l| \leq L} u_{l} e(\alpha l) .
$$

Then, whenever $1 \leq Q \leq N^{1 / 2}$ one has

$$
\int_{\mathfrak{M}(Q)}\left|f_{2}(\alpha) U(\alpha)\right|^{2} \mathrm{~d} \alpha \ll U(0)^{2} N^{\varepsilon}+Q N^{\varepsilon} \int_{0}^{1}|U(\alpha)|^{2} \mathrm{~d} \alpha .
$$

Proof. Let $\alpha \in \mathfrak{I}(q, a)$, and write $\alpha=(a / q)+\beta$. Then, by (2.2), 2.3) and (2.4), we see that

$$
|f(\alpha)|^{2} \ll N(q+N q|\beta|)^{-1}+q^{\varepsilon}(q+N q|\beta|) \ll N^{1+\varepsilon}(q+N q|\beta|)^{-1}
$$

Hence, at least when $Q \leq \frac{1}{2} N^{1 / 2}$, we may apply [2] Lemma 1] with $\Psi(\alpha)=|U(\alpha)|^{2}$ and

$$
\psi_{h}=\sum_{l-l^{\prime}=h} u_{l} u_{l^{\prime}}
$$

to see that

$$
\int_{\mathfrak{M}(Q)}\left|f_{2}(\alpha) U(\alpha)\right|^{2} \mathrm{~d} \alpha \ll N^{\varepsilon}\left(Q \psi_{0}+\sum_{h} \psi_{h}\right) .
$$

By orthogonality,

$$
\psi_{0}=\int_{0}^{1}|U(\alpha)|^{2} \mathrm{~d} \alpha
$$

while the $\psi_{h}$ sum to $|U(0)|^{2}$, as required. When $\frac{1}{2} N^{1 / 2}<Q \leq N^{1 / 2}$, the now fuzzy ends of the intervals defining the major arcs obstruct a direct reference to [2, Lemma 1], yet an inspection of the proof of this lemma shows that the situation is entirely covered by the argument given there, and the bound (2.13) follows in the same way.

We now give some applications of Lemma 1, in the order they appear in the course of the argument. We take $U(\alpha)=f_{4}(\alpha) g_{6}(\alpha)$ and note that by orthogonality and an 
elementary divisor argument one has

$$
\int_{0}^{1}\left|f_{4}(\alpha) g_{6}(\alpha)\right|^{2} \mathrm{~d} \alpha \ll P_{4} P_{6} .
$$

Hence, when $N^{5 / 12} \leq Q \leq N^{1 / 2}$, Lemma 1 yields

$$
\int_{\mathfrak{M}(Q)}\left|f_{2}(\alpha) f_{4}(\alpha) g_{6}(\alpha)\right|^{2} \mathrm{~d} \alpha \ll N^{\varepsilon} Q P_{4} P_{6}
$$

For comparison, and to substantiate a comment made in the introduction, note here that

$$
\int_{0}^{1}\left|f_{2}(\alpha) f_{4}(\alpha) g_{6}(\alpha)\right|^{2} \mathrm{~d} \alpha \gg P_{2} P_{4} P_{6},
$$

as one readily confirms by counting the diagonal solutions of the underlying diophantine equation. The bound 2.15) saves over this, and the saving increases with shrinking values of $Q$. This feature is typical for a traditional pruning estimate.

Next, take $Q=N^{5 / 12}$ and $U(\alpha)=f_{8}(\alpha)^{6}$. Then, by Schwarz's inequality and Hua's lemma [14, Lemma 2.5], one finds that

$$
\int_{0}^{1}\left|f_{8}(\alpha)\right|^{12} \mathrm{~d} \alpha \leq\left(\int_{0}^{1}\left|f_{8}(\alpha)\right|^{8} \mathrm{~d} \alpha\right)^{1 / 2}\left(\int_{0}^{1}\left|f_{8}(\alpha)\right|^{16} \mathrm{~d} \alpha\right)^{1 / 2} \ll P_{8}^{17 / 2+\varepsilon} \ll P_{8}^{12} Q^{-1} .
$$

Hence, Lemma 1 reveals

$$
\int_{\mathfrak{M}\left(N^{5 / 12}\right)}\left|f_{2}(\alpha) f_{8}(\alpha)^{6}\right|^{2} \mathrm{~d} \alpha \ll P_{8}^{12+\varepsilon} .
$$

With $Q$ as before we now choose $U(\alpha)=g_{6}(\alpha)^{2} f_{8}(\alpha)$. Here Hölder's inequality gives

$$
\int_{0}^{1}\left|g_{6}^{2} f_{8}\right|^{2} \mathrm{~d} \alpha \leq\left(\int_{0}^{1}\left|g_{6}\right|^{6} \mathrm{~d} \alpha\right)^{2 / 3}\left(\int_{0}^{1}\left|f_{8}\right|^{4} \mathrm{~d} \alpha\right)^{1 / 6}\left(\int_{0}^{1}\left|f_{8}\right|^{8} \mathrm{~d} \alpha\right)^{1 / 6} .
$$

Again, we use Hua's lemma to estimate the second and third factor while 2.9 estimates the first. This gives

$$
\int_{0}^{1}\left|g_{6}^{2} f_{8}\right|^{2} \mathrm{~d} \alpha \ll N^{\varepsilon}\left(P_{6}^{3+1 / 11}\right)^{2 / 3}\left(P_{8}^{2}\right)^{1 / 6}\left(P_{8}^{5}\right)^{1 / 6} \ll P_{6}^{4} P_{8}^{2} N^{-5 / 12},
$$

as one readily checks. Therefore, Lemma 1 shows that

$$
\int_{\mathfrak{M}\left(N^{5 / 12}\right)}\left|f_{2}(\alpha) g_{6}(\alpha)^{2} f_{8}(\alpha)\right|^{2} \mathrm{~d} \alpha \ll P_{6}^{4} P_{8}^{2} N^{\varepsilon},
$$

and from 2.16 and Schwarz's inequality we finally deduce that

$$
\int_{\mathfrak{M}\left(N^{5 / 12}\right)}\left|f_{2}(\alpha) g_{6}(\alpha)\right|^{2}\left|f_{8}(\alpha)\right|^{7} \mathrm{~d} \alpha \ll P_{6}^{2} P_{8}^{7} N^{\varepsilon} .
$$

This is the second of our pruning estimates. The odd power attached to $f_{8}$ is a curious feature, preventing a direct application of Lemma 1. The bound (2.17) is the best possible, save for the factor $N^{\varepsilon}$.

3. Unconventional attack. We launch our attack by preparing the ground for an application of the Hardy-Littlewood method. Let

$$
F(\alpha)=f_{2}(\alpha) f_{4}(\alpha) g_{6}(\alpha) f_{8}(\alpha) .
$$


Then, whenever $n \leq N$, the integral

$$
\varrho(n, N)=\int_{\mathfrak{U}} F(\alpha) e(-\alpha n) \mathrm{d} \alpha
$$

counts the solutions of 1.3 with $x_{j} \in \mathbb{N}$ and $x_{3} \in \mathcal{A}\left(\frac{1}{2} P_{6}, P_{6}^{\eta}\right)$. Hence it is evident that

$$
r(n) \geq \varrho(n, N) \quad(n \leq N) .
$$

There is quite some flexibility in the definition of major and minor arcs, and we take

$$
\mathfrak{M}=\mathfrak{M}\left(N^{1 / 13}\right), \quad \mathfrak{m}=\mathfrak{U} \backslash \mathfrak{M} .
$$

Then

$$
\varrho(n, N)=\varrho_{\mathfrak{M}}(n, N)+\varrho_{\mathfrak{m}}(n, N)
$$

where, for a measurable set $\mathfrak{a} \subset \mathfrak{U}$, we wrote

$$
\varrho_{\mathfrak{a}}(n, N)=\int_{\mathfrak{a}} F(\alpha) e(-\alpha n) \mathrm{d} \alpha .
$$

The principal goal of this section is to show that $\varrho_{\mathfrak{m}}(n, N)$ is small in mean square. In the interest of brevity, we put

$$
\theta=\frac{1}{24}-\frac{\delta(5)}{36}=0.021536 \ldots
$$

and note that $\theta>2 / 93$.

LEMMA 2. One has

$$
\sum_{n \leq N}\left|\varrho_{\mathfrak{m}}(n, N)\right|^{2} \ll N^{13 / 12-\theta+\varepsilon} .
$$

The proof will occupy the remainder of this section. This part is, perhaps, the most original aspect of this paper. We begin by noting that $\mathfrak{m}=\mathfrak{M}\left(N^{1 / 2}\right) \backslash \mathfrak{M}\left(N^{1 / 13}\right)$. We write $\mathfrak{N}(Q)=\mathfrak{M}(2 Q) \backslash \mathfrak{M}(Q)$. Then $\mathfrak{m}$ is covered by no more than $\log N$ sets $\mathfrak{N}(Q)$ with $N^{1 / 13} \leq Q \leq \frac{1}{2} N^{1 / 2}$. By Bessel's inequality, we see that there is a value of $Q$ in this range with

$$
\sum_{n \leq N}\left|\varrho_{\mathfrak{m}}(n, N)\right|^{2} \leq \int_{\mathfrak{m}}|F(\alpha)|^{2} \mathrm{~d} \alpha \leq(\log N) \int_{\mathfrak{N}(Q)}|F(\alpha)|^{2} \mathrm{~d} \alpha .
$$

We consider cases, depending on the size of $Q$. Put $Q=N^{\omega}$. Then $\frac{1}{13} \leq \omega<\frac{1}{2}$. The range $\frac{5}{12} \leq \omega<\frac{1}{2}$ is considered first. We choose a parameter $X$ with $1 \leq X \leq P_{6}$ and split $\mathfrak{N}(Q)$ into the sets

$$
\mathcal{B}=\left\{\alpha \in \mathfrak{N}(Q):\left|g_{6}(\alpha)\right| \geq P_{6} X^{-1}\right\} \quad \text { and } \quad \mathcal{C}=\mathfrak{N}(Q) \backslash \mathcal{B} .
$$

Then, since $g_{6}(\alpha) \ll P_{6} X^{-1}$ holds on $\mathcal{C}$, we apply Schwarz's inequality in the form

$$
\int_{\mathcal{C}}|F|^{2} \mathrm{~d} \alpha \leq\left(\int_{0}^{1}\left|f_{2} f_{4} f_{8}^{2}\right|^{2} \mathrm{~d} \alpha\right)^{1 / 2}\left(\int_{\mathfrak{M}(2 Q)}\left|f_{2} f_{4} g_{6}\right|^{2} \mathrm{~d} \alpha\right)^{1 / 2} \sup _{\alpha \in \mathcal{C}}\left|g_{6}(\alpha)\right|,
$$

to conclude from 2.8 and 2.15 that

$$
\int_{\mathcal{C}}|F|^{2} \mathrm{~d} \alpha \ll\left(N^{1+\varepsilon}\right)^{1 / 2}\left(Q P_{4} P_{6}\right)^{1 / 2} P_{6} X^{-1} \ll N^{7 / 8+\varepsilon} Q^{1 / 2} X^{-1} .
$$


On the set $\mathcal{B}$ we explore the fact that $g_{6}$ is large. By 2.9 ,

$$
\int_{\mathcal{B}}\left|g_{6}\right|^{8} \mathrm{~d} \alpha \leq X^{2} P_{6}^{-2} \int_{0}^{1}\left|g_{6}\right|^{10} \mathrm{~d} \alpha \ll X^{2} P_{6}^{3+\delta(5)+\varepsilon} .
$$

Note that when $X$ is not too large this is considerably stronger than the bound provided by 2.9 with $s=4$. This amplification effect is the pivotal element in the pruning procedure. By Hölder's inequality,

$\int_{\mathcal{B}}|F|^{2} \mathrm{~d} \alpha \leq\left(\int_{0}^{1}\left|f_{2} f_{4} f_{8}^{2}\right|^{2} \mathrm{~d} \alpha\right)^{1 / 2}\left(\int_{0}^{1}\left|f_{2} f_{4}^{2}\right|^{2} \mathrm{~d} \alpha\right)^{1 / 4}\left(\int_{\mathcal{B}}\left|g_{6}\right|^{8} \mathrm{~d} \alpha\right)^{1 / 4} \sup _{\alpha \in \mathfrak{N}(Q)}\left|f_{2}(\alpha)\right|^{1 / 2}$.

By 2.14, for $\alpha \in \mathfrak{N}(Q)$ one has $\left|f_{2}(\alpha)\right|^{2} \ll N^{1+\varepsilon} Q^{-1}$. From 2.8 and (3.8) we infer

$$
\int_{\mathcal{B}}|F|^{2} \mathrm{~d} \alpha \ll N^{3 / 4+\varepsilon}\left(X^{2} P_{6}^{3+\delta(5)}\right)^{1 / 4} N^{1 / 4} Q^{-1 / 4} \ll N^{9 / 8+\delta(5) / 24+\varepsilon} X^{1 / 2} Q^{-1 / 4} .
$$

We balance (3.9) and (3.7), keeping in mind the condition that $1 \leq X \leq P_{6}$. With $X=N^{\xi}$ the far right of (3.7) becomes $N^{\Theta+\varepsilon}$ where

$$
\Theta=\frac{7}{8}+\frac{\omega}{2}-\xi
$$

If we write the right hand side of $(3.9)$ as $N^{\Lambda+\varepsilon}$, then $\Lambda=\frac{9}{8}+\frac{\delta(5)}{24}+\frac{1}{2} \xi-\frac{1}{4} \omega$. We choose

$$
\xi=\frac{\omega}{2}-\frac{1}{6}-\frac{\delta(5)}{36}
$$

to arrange that $\Theta=\Lambda$. From $\frac{5}{12} \leq \omega<\frac{1}{2}$ and the numerical value for $\delta(5)$ one quickly confirms that $\frac{1}{100} \leq \xi \leq \frac{1}{12}$, an admissible choice. With this value of $\xi$ we see from (3.10) and 3.5 that

$$
\Theta=\frac{7}{8}+\frac{1}{6}+\frac{\delta(5)}{36}=\frac{13}{12}-\theta .
$$

Consequently, the estimates 3.7 and 3.9 add to

$$
\int_{\mathfrak{N}(Q)}|F(\alpha)|^{2} \mathrm{~d} \alpha \ll N^{13 / 12-\theta+\varepsilon} .
$$

We now turn to the case where $\frac{1}{6} \leq \omega<\frac{5}{12}$, and more precisely, where $2 Q \leq N^{5 / 12}$, as we may assume. Then $\mathfrak{N}(Q) \subset \mathfrak{M}\left(N^{5 / 12}\right)$. Let

$$
\mathcal{D}=\left\{\alpha \in \mathfrak{N}(Q):\left|f_{8}(\alpha)\right|^{2} \leq P_{8}^{2} N^{-\theta}\right\}, \quad \mathcal{E}=\mathfrak{N}(Q) \backslash \mathcal{D} .
$$

The treatment of the set $\mathcal{D}$ is straightforward. One imports 2.15 to conclude that

$$
\int_{\mathcal{D}}|F(\alpha)|^{2} \mathrm{~d} \alpha \leq P_{8}^{2} N^{-\theta} \int_{\mathfrak{M}\left(N^{5 / 12}\right)}\left|f_{2} f_{4} g_{6}\right|^{2} \mathrm{~d} \alpha \ll N^{13 / 12-\theta+\varepsilon} .
$$

When $\alpha \in \mathcal{E}$ one has $\left|f_{8}(\alpha)\right|^{2}>P_{8}^{2} N^{-\theta}$, and therefore,

$$
\int_{\mathcal{E}}\left|f_{2} g_{6} f_{8}\right|^{2} \mathrm{~d} \alpha<N^{5 \theta / 2} P_{8}^{-5} \int_{\mathfrak{N}(Q)}\left|f_{2} g_{6}\right|^{2}\left|f_{8}\right|^{7} \mathrm{~d} \alpha .
$$

From 2.17) we deduce that

$$
\int_{\mathcal{E}}\left|f_{2} g_{6} f_{8}\right|^{2} \mathrm{~d} \alpha \ll P_{6}^{2} P_{8}^{2} N^{5 \theta / 2+\varepsilon} .
$$


Now take $k=4$ in 2.2 and use (2.3) and 2.4 to confirm that, for $\alpha \in \mathfrak{N}(Q)$, one has

$$
\left|f_{4}(\alpha)\right|^{2} \ll N^{1 / 2} Q^{-1 / 2}+Q^{1+\varepsilon} \ll N^{5 / 12+\varepsilon}=P_{4}^{2} N^{\varepsilon-1 / 12} .
$$

Since $\frac{5}{2} \theta-\frac{1}{12}<-\theta$ we conclude from 3.13 and 3.14 that

$$
\int_{\mathcal{E}}|F(\alpha)|^{2} \mathrm{~d} \alpha \ll N^{13 / 12-\theta+\varepsilon} .
$$

In view of 3.12 we now see that 3.11 also holds in the range $\frac{1}{6} \leq \omega \leq \frac{5}{12}$.

This leaves the range $\frac{1}{13} \leq \omega \leq \frac{1}{6}$. A straightforward application of Lemma 1 yields

$$
\int_{\mathfrak{N}(Q)}\left|f_{2}(\alpha) g_{6}(\alpha)\right|^{2} \mathrm{~d} \alpha \ll P_{6}^{2+\varepsilon}
$$

while the first inequality in 3.14 shows that $\left|f_{4}(\alpha)\right|^{2} \ll P_{4}^{2} N^{-1 / 26}$ whenever $\alpha \in \mathfrak{N}(Q)$. The trivial estimate for $f_{8}$ now suffices to conclude that

$$
\int_{\mathfrak{N}(Q)}|F(\alpha)|^{2} \mathrm{~d} \alpha \ll N^{13 / 12-1 / 26+\varepsilon},
$$

a bound much superior to 3.11. Lemma 2 now follows from 3.6 .

4. Decoupling. In this section we begin with the major arc analysis, by separating the singular series from the singular integral as far as one can get without entering the distribution of smooth numbers. The decoupling will take several steps. We begin by introducing more notation. For $1 \leq a \leq q \leq N^{1 / 13}$ and $(a, q)=1$ let

$$
\mathfrak{P}(q, a)=\left\{\alpha:|\alpha-(a / q)| \leq N^{-1 / 3}\right\} .
$$

These intervals are disjoint, at least when $N$ is large. We write $\mathfrak{P}$ for their union, and for $k \geq 2$ we define $f_{k}^{*}: \mathfrak{P} \rightarrow \mathbb{C}$ by

$$
f^{*}(\alpha)=q^{-1} S_{k}(q, a) v_{k}(\alpha-(a / q)) \quad \text { whenever } \quad \alpha \in \mathfrak{P}(q, a) .
$$

Let $\mathfrak{A} \subset \mathfrak{P}$ be measurable and put

$$
\varrho_{\mathfrak{A}}^{*}(n, N)=\int_{\mathfrak{A}} f_{2}^{*}(\alpha) f_{4}^{*}(\alpha) f_{8}^{*}(\alpha) g_{6}(\alpha) e(-\alpha n) \mathrm{d} \alpha .
$$

Classical major arc work provides us with the following estimate.

LEMMA 3. One has

$$
\sum_{n \leq N}\left|\varrho_{\mathfrak{M}}(n, N)-\varrho_{\mathfrak{P}}^{*}(n, N)\right|^{2} \ll N^{13 / 12-1 / 26+\varepsilon} .
$$

Proof. The main difficulty is with the approximation of $\varrho_{\mathfrak{M}}(n, N)$ by $\varrho_{\mathfrak{M}}^{*}(n, N)$. By 3.1 , (3.2) and 4.1), followed by Bessel's inequality and a trivial bound for $g_{6}$, one finds that

$$
\begin{aligned}
\sum_{n \leq N}\left|\varrho_{\mathfrak{M}}(n, N)-\varrho_{\mathfrak{M}}^{*}(n, N)\right|^{2} & \ll \int_{\mathfrak{M}}\left|f_{2} f_{4} f_{8}-f_{2}^{*} f_{4}^{*} f_{8}^{*}\right|^{2}\left|g_{6}\right|^{2} \mathrm{~d} \alpha \\
& \ll P_{6}^{2} \int_{\mathfrak{M}}\left|f_{2} f_{4} f_{8}-f_{2}^{*} f_{4}^{*} f_{8}^{*}\right|^{2} \mathrm{~d} \alpha .
\end{aligned}
$$


Further, by 2.2 we see that $f_{k}-f_{k}^{*} \ll N^{1 / 26+\varepsilon}$ holds uniformly on $\mathfrak{M}$. Hence, using trivial bounds frequently, one first finds that

$$
f_{2} f_{4}=\left(f_{2}-f_{2}^{*}\right) f_{4}+f_{2}^{*}\left(f_{4}-f_{4}^{*}\right)+f_{2}^{*} f_{4}^{*}=f_{2}^{*} f_{4}^{*}+O\left(P_{2} N^{1 / 26+\varepsilon}\right),
$$

and then, since the measure of $\mathfrak{M}$ is $O\left(N^{-11 / 13}\right)$, multiplication of the preceding line with $f_{8}$ suffices to confirm the bound

$$
\int_{\mathfrak{M}}\left|f_{2} f_{4}-f_{2}^{*} f_{4}^{*}\right|^{2}\left|f_{8}\right|^{2} \mathrm{~d} \alpha \ll P_{2}^{2} P_{8}^{2} N^{1 / 13+\varepsilon} \int_{\mathfrak{M}} \mathrm{d} \alpha \ll P_{8}^{2} N^{3 / 13+\varepsilon} .
$$

Next, we first observe that

$$
\int_{\mathfrak{M}}\left|f_{2}^{*} f_{4}^{*}\right|^{2}\left|f_{8}-f_{8}^{*}\right|^{2} \mathrm{~d} \alpha \ll N^{1 / 13+\varepsilon} \int_{\mathfrak{M}}\left|f_{2}^{*} f_{4}^{*}\right|^{2} \mathrm{~d} \alpha,
$$

and then we use $2.3,2.4,2.5$ and 2.6 to confirm that

$$
\int_{\mathfrak{M}}\left|f_{2}^{*} f_{4}^{*}\right|^{2} \mathrm{~d} \alpha \leq P_{2}^{2} P_{4}^{2} \sum_{q \leq P_{8}} \sum_{\substack{a=1 \\(a, q)=1}}^{q} q^{-1} \kappa_{4}(q)^{2} \int_{-\infty}^{\infty}(1+N|\beta|)^{-3 / 2} \mathrm{~d} \beta \ll P_{4}^{2} \sum_{q \leq P_{8}} \kappa_{4}(q)^{2} .
$$

We invoke 2.7 to bound the sum on the far right, and then conclude that

$$
\int_{\mathfrak{M}}\left|f_{2}^{*} f_{4}^{*}\right|^{2}\left|f_{8}-f_{8}^{*}\right|^{2} \mathrm{~d} \alpha \ll P_{4}^{2} N^{1 / 13+\varepsilon} .
$$

This we combine with 4.3 and apply the elementary inequality

$$
|u+v|^{2} \leq 2|u|^{2}+2|v|^{2}
$$

to estimate the integral in the lower line of 4.2 , thus producing the bound

$$
\sum_{n \leq N}\left|\varrho_{\mathfrak{M}}(n, N)-\varrho_{\mathfrak{M}}^{*}(n, N)\right|^{2} \ll N
$$

The next step is to compare $\varrho_{\mathfrak{P}}^{*}(n, N)$ with $\varrho_{\mathfrak{M}}^{*}(n, N)$. Their difference is $\varrho_{\mathfrak{P} \backslash \mathfrak{M}}^{*}(n, N)$, so that Bessel's inequality yields

$$
\sum_{n \leq N}\left|\varrho_{\mathfrak{M}}^{*}(n, N)-\varrho_{\mathfrak{P}}^{*}(n, N)\right|^{2} \leq \int_{\mathfrak{P} \backslash \mathfrak{M}}\left|f_{2}^{*} f_{4}^{*} f_{8}^{*} g_{6}\right|^{2} \mathrm{~d} \alpha \leq P_{6}^{2} \int_{\mathfrak{P} \backslash \mathfrak{M}}\left|f_{2}^{*} f_{4}^{*} f_{8}^{*}\right|^{2} \mathrm{~d} \alpha .
$$

By 2.3, 2.4 and 2.5, and then (2.7), we see

$$
\begin{aligned}
\int_{\mathfrak{P} \backslash \mathfrak{M}}\left|f_{2}^{*} f_{4}^{*} f_{8}^{*}\right|^{2} \mathrm{~d} \alpha & \ll P_{2}^{2} P_{4}^{2} P_{8}^{2} \sum_{q \leq P_{13}} q^{-1 / 2} \kappa_{8}(q)^{2} \int_{P_{13} /(q N)}^{\infty}(1+N|\beta|)^{-7 / 4} \mathrm{~d} \beta \\
& \ll P_{4}^{2} P_{8}^{2} \sum_{q \leq P_{13}} P_{13}^{-3 / 4} q^{1 / 4} \kappa_{8}(q)^{2} \ll\left(P_{4} P_{8}\right)^{2} P_{13}^{\varepsilon-1 / 2},
\end{aligned}
$$

and it follows that

$$
\sum_{n \leq N}\left|\varrho_{\mathfrak{M}}^{*}(n, N)-\varrho_{\mathfrak{P}}^{*}(n, N)\right|^{2} \ll N^{13 / 12-1 / 26+\varepsilon}
$$

We combine this with 4.5, and apply 4.4. The lemma follows. 
We are ready to perform the decoupling. It is convenient to present $\varrho_{\mathfrak{P}}^{*}(n, N)$ in another way. Let $q \in \mathbb{N}, m \in \mathbb{Z}$, and let $Y$ be a positive real number. Define

$$
\begin{gathered}
A(q, m)=q^{-3} \sum_{\substack{a=1 \\
(a, q)=1}}^{q} S_{2}(q, a) S_{4}(q, a) S_{8}(q, a) e\left(-\frac{a m}{q}\right), \\
J(N, Y, m)=\int_{-Y}^{Y} v_{2}(\beta) v_{4}(\beta) v_{8}(\beta) e(-\beta m) \mathrm{d} \beta .
\end{gathered}
$$

Then, by 2.1), 2.10) and 4.1),

$$
\varrho_{\mathfrak{P}}^{*}(n, N)=\sum_{y \in \mathcal{A}\left(\frac{1}{2} P_{6}, P_{6}^{\eta}\right)} \sum_{q \leq P_{13}} A\left(q, n-y^{6}\right) J\left(N, N^{-1 / 3}, n-y^{6}\right) .
$$

Note that $A(q, m)$ is independent of $N$ while $J$ is independent of $q$. We wish to remove the dependence on $N$ also from the $J$-integral, at least for $m<N$. We therefore bring in the function $J\left(N, \frac{1}{2}, m\right)=J(N, m)$, say, and compare 4.8 with

$$
\varrho^{\dagger}(n, N)=\sum_{y \in \mathcal{A}\left(\frac{1}{2} P_{6}, P_{6}^{\eta}\right)} \sum_{q \leq P_{13}} A\left(q, n-y^{6}\right) J\left(N, n-y^{6}\right) .
$$

The difference $\varrho^{\dagger}(n, N)-\varrho_{\mathfrak{P}}^{*}(n, N)$ is then given by

$$
\sum_{y \in \mathcal{A}\left(\frac{1}{2} P_{6}, P_{6}^{\eta}\right)} \sum_{q \leq P_{13}} A\left(q, n-y^{6}\right) \int_{N^{-1 / 3} \leq|\beta| \leq 1 / 2} v_{2}(\beta) v_{4}(\beta) v_{8}(\beta) e\left(\beta\left(y^{6}-n\right)\right) \mathrm{d} \beta,
$$

so that Cauchy's inequality delivers

$$
\begin{aligned}
& \left|\varrho^{\dagger}(n, N)-\varrho_{\mathfrak{P}}^{*}(n, N)\right|^{2} \\
& \quad \leq \mathrm{A} \sum_{y \leq P_{6}}\left|\int_{N^{-1 / 3} \leq|\beta| \leq 1 / 2} v_{2}(\beta) v_{4}(\beta) v_{8}(\beta) e\left(\beta y^{6}\right) e(-\beta n) \mathrm{d} \beta\right|^{2}
\end{aligned}
$$

where

$$
\mathrm{A}=\sum_{y \in \mathcal{A}\left(\frac{1}{2} P_{6}, P_{6}^{\eta}\right)}\left|\sum_{q \leq P_{13}} A\left(q, n-y^{6}\right)\right|^{2} .
$$

By (4.6), (2.4) and (2.6), the inequality

$$
A(q, m) \ll q^{1 / 2} \kappa_{4}(q) \kappa_{8}(q)
$$

holds uniformly $m$, and therefore, by 2.7 and Cauchy's inequality, the estimate

$$
\sum_{q \leq Q}|A(q, m)| \ll Q^{1 / 2+\varepsilon}
$$

holds uniformly in $m$ as well. This shows $\mathrm{A} \ll P_{6} P_{13}^{1+\varepsilon}$. Further, Bessel's inequality yields

$$
\sum_{n \leq N}\left|\int_{N^{-1 / 3} \leq|\beta| \leq 1 / 2} v_{2}(\beta) v_{4}(\beta) v_{8}(\beta) e\left(\beta y^{6}\right) e(-\beta n) \mathrm{d} \beta\right|^{2} \leq \int_{N^{-1 / 3} \leq|\beta| \leq 1 / 2}\left|v_{2} v_{4} v_{8}\right|^{2} \mathrm{~d} \beta,
$$

and here, by 2.3 , the right hand side does not exceed

$$
\ll\left(P_{2} P_{4} P_{8}\right)^{2} \int_{N^{-1 / 3}}^{\infty}(1+N|\beta|)^{-7 / 4} \mathrm{~d} \beta \ll P_{4}^{2} P_{8}^{2} N^{-1 / 2} .
$$


Collecting together, summation of 4.10 over $n$ delivers the desired estimate

$$
\sum_{n \leq N}\left|\varrho_{\mathfrak{P}}^{*}(n, N)-\varrho^{\dagger}(n, N)\right|^{2} \ll\left(P_{4} P_{6} P_{8}\right)^{2} N^{1 / 13-1 / 2} \ll N .
$$

The expression 4.9 simplifies further. Indeed, by 4.7), 2.1 and orthogonality, we have

$$
J(N, m)=\frac{1}{64} \sum_{\substack{1 \leq l_{2}, l_{4}, l_{8} \leq N \\ l_{2}+l_{4}+l_{8}=m}} l_{2}^{-1 / 2} l_{4}^{-3 / 4} l_{8}^{-7 / 8} .
$$

Note here that for $1 \leq m \leq N$ the sum $J(m, N)$ is independent of $N$. We suppose that $\frac{1}{2} N<n \leq N$ and take $m=n-y^{6}$ with $1 \leq y \leq \frac{1}{2} P_{6}$. Then $m>\frac{3}{8} N$, and two applications of [14, Lemma 2.9] show that

$$
J(N, m)=\Gamma m^{-1 / 8}+O\left(m^{-1 / 4}\right)
$$

where $\Gamma=\Gamma\left(\frac{3}{2}\right) \Gamma\left(\frac{5}{4}\right) \Gamma\left(\frac{9}{8}\right) / \Gamma\left(\frac{7}{8}\right)$. We therefore bring in the sum

$$
\sigma(n, N)=\sum_{y \in \mathcal{A}\left(\frac{1}{2} P_{6}, P_{6}^{\eta}\right)} \sum_{q \leq P_{13}} A\left(q, n-y_{6}\right)\left(n-y^{6}\right)^{-1 / 8} .
$$

where again we suppose that $\frac{1}{2} N<n \leq N$. According to the penultimate display and 4.9 , there are numbers $E(m)$ with $E(m) \ll N^{-1 / 4}$, and such that

$$
\varrho^{\dagger}(n, N)-\Gamma \sigma(n, N)=\sum_{y \in \mathcal{A}\left(\frac{1}{2} P_{6}, P_{6}^{\eta}\right)} \sum_{q \leq P_{13}} A\left(q, n-y^{6}\right) E\left(n-y^{6}\right) .
$$

Hence, by 4.12 and elementary estimates, whenever $\frac{1}{2} N<n \leq N$, one has

$$
\varrho^{\dagger}(n, N)-\Gamma \sigma(n, N) \ll P_{6} P_{13}^{1 / 2+\varepsilon} N^{-1 / 4},
$$

and we conclude that

$$
\sum_{N / 2<n \leq N}\left|\varrho^{\dagger}(n, N)-\Gamma \sigma(n, N)\right|^{2} \ll N .
$$

It is time to sum up the initial circle method work, performed in mean square over $n$. Indeed, on combining 4.15 with 4.13 and the results from Lemmata 2 and 3 through (3.4) and repeated use of (4.4), we may conclude as follows.

LEMMA 4. One has

$$
\sum_{N / 2<n \leq N}|\varrho(n, N)-\Gamma \sigma(n, N)|^{2} \ll N^{13 / 12-\theta+\varepsilon} .
$$

5. Local interlude. In this section we gather local information encoded by $A(q, m)$. The precise estimates that we obtain for $A(q, m)$ are then applied to the cognate sum

$$
B(q, n)=\sum_{b=1}^{q}\left|A\left(q, n-b^{6}\right)\right| .
$$

Lemma 5. Suppose that $m, n \in \mathbb{Z}$. Then $A(q, m)$ and $B(q, n)$ are multiplicative in $q$. 
Proof. The argument of proof of [14, Lemma 2.11] yields the multiplicativity of $A(q, m)$. Also, by 4.6), we see that $A(q, m)$ has period $q$ in $m$. Therefore, whenever $q=q_{1} q_{2}$ with $\left(q_{1}, q_{2}\right)=1$, we may substitute $b=b_{1} q_{2}+b_{2} q_{1}$ in (5.1) to verify that

$$
B(q, n)=\sum_{b_{1}=1}^{q_{1}} \sum_{b_{2}=1}^{q_{2}}\left|A\left(q_{1}, n-\left(b_{1} q_{2}\right)^{6}\right) A\left(q_{2}, n-\left(b_{2} q_{1}\right)^{6}\right)\right| .
$$

Here $b_{1} q_{2}$ runs over a complete set of residues modulo $q_{1}$ as $b_{1}$ does, so that the sum of $\left|A\left(q_{1}, n-\left(b_{1} q_{2}\right)^{6}\right)\right|$ over $b_{1}$ is $B\left(q_{1}, n\right)$. By symmetry, it follows that $B(q, n)$ is multiplicative.

By Lemma 5 it suffices to consider the case where $q$ is a power of a prime $p$. In addressing this case, we frequently require the reduction formula

$$
S_{k}\left(p^{u+k}, a\right)=p^{k-1} S_{k}\left(p^{u}, a\right)
$$

that is valid for odd primes $p$ with $p \nmid a$, all $u \geq 0$ and $k=2,4,8$. If, in addition, one has $2 \leq u \leq k$, then

$$
S_{k}\left(p^{u}, a\right)=p^{u-1}
$$

When $p=2,5.2$ still holds for all $u \geq 2$. Later we also require 5.2 and $(5.3)$ for $k=6$ where these statements hold for primes $p>3$. When $p=2$ or 3 we have 5.2 for $u \geq 2$ $(p=2)$ or $u \geq 1(p=3)$. All of this are special cases of [14, Lemma 4.4].

The case $u=1$ is more subtle. With $\chi$ a Dirichlet character modulo $p$, let

$$
\tau(\chi)=\sum_{a=1}^{p-1} \chi(a) e(a / p)
$$

denote the Gauß sum and recall that $|\tau(\chi)|^{2}=p$ whenever $\chi$ is non-principal. Let $\mathrm{X}_{k}$ denote the set of non-principal characters $\chi$ modulo $p$ where $\chi^{k}$ is principal. There are $(k, p-1)-1$ such characters. Lemma 4.3 of Vaughan [14] asserts that

$$
S_{k}(p, a)=\sum_{\chi \in \mathrm{X}_{k}} \chi(a) \tau(\bar{\chi}) \quad(p \text { odd, } p \nmid a, k=2,4,6,8) .
$$

Since $k$ is even, the set $\mathrm{X}_{k}$ contains the Legendre symbol, and this is the only element of $\mathrm{X}_{2}$. Hence, by 5.4 with $a=1$, one finds that $S(p, 1)$ is the Gauß sum for the Legendre symbol. Then, using (5.4) again, we obtain

$$
S_{2}(p, a)=\left(\frac{a}{p}\right) S_{2}(p, 1) \quad(p \nmid a) .
$$

Lemma 6. Let $m \in \mathbb{Z}$, let $u \in \mathbb{N}$, and let $p$ be an odd prime. Then $A\left(p^{8+u}, m\right)=0$ unless $p^{8} \mid m$ in which case one has $A\left(p^{8+u}, m\right)=p A\left(p^{u}, m / p^{8}\right)$. For $p=2$ this remains valid for all $u \geq 2$. 
Proof. By 4.6 and 5.2 ,

$$
\begin{aligned}
A\left(p^{8+u}, m\right) & =p^{-7-3 u} \sum_{\substack{a=1 \\
p \nmid a}}^{p^{8+u}} S_{2}\left(p^{u}, a\right) S_{4}\left(p^{u}, a\right) S_{8}\left(p^{u}, a\right) e\left(-\frac{a m}{p^{8+u}}\right) \\
& =p^{-7-3 u} \sum_{\substack{b=1 \\
p \nmid b}}^{p^{u}} \sum_{c=1}^{p^{8}} S_{2}\left(p^{u}, b\right) S_{4}\left(p^{u}, b\right) S_{8}\left(p^{u}, b\right) e\left(-\frac{\left(b+p^{u} c\right) m}{p^{8+u}}\right) .
\end{aligned}
$$

The sum over $c$ vanishes unless $p^{8} \mid m$, and in that event it produces a factor $p^{8}$. The lemma follows.

Within the proofs of the next lemmata we will encounter Ramanujan's sum

$$
c_{q}(m)=\sum_{\substack{a=1 \\(a, q)=1}}^{q} e\left(-\frac{a m}{q}\right)
$$

and we require the explicit evaluations

$$
c_{p^{u}}(m)=0\left(p^{u-1} \nmid m\right), \quad c_{p^{u}}(m)=-p^{u-1}\left(p^{u-1} \| m\right), \quad c_{p^{u}}(m)=\varphi\left(p^{u}\right)\left(p^{u} \mid m\right)
$$

that are valid for all primes $p$, all $u \geq 1$ ([8, Theorem 272]).

Lemma 7. Let $m \in \mathbb{Z}$, and let $p$ be a prime. Then $A(p, m) \ll p^{-1}(p, m)^{1 / 2}$.

Proof. This is trivial for $p=2$ so we suppose that $p$ is odd. By (4.6), (5.4) and (5.5),

$$
A(p, m)=p^{-3} S_{2}(p, 1) \sum_{\chi_{4} \in \mathrm{X}_{4}} \sum_{\chi_{8} \in \mathrm{X}_{8}} \tau\left(\bar{\chi}_{4}\right) \tau\left(\bar{\chi}_{8}\right) \sum_{a=1}^{p-1}\left(\frac{a}{p}\right) \chi_{4} \chi_{8}(a) e\left(-\frac{a m}{p}\right) .
$$

It will be convenient to denote, temporarily, the Legendre symbol modulo $p$ by $\chi_{2}$.

First suppose that $p \mid m$. Then the sum over $a$ is trivially smaller than $p$ in modulus, and the estimate for the Gauß sum yields the crude bound $|A(p, m)| \leq 21 p^{-1 / 2}$. Next suppose that $p \nmid m$. If the character $\chi_{2} \chi_{4} \chi_{8}$ is principal, then the sum over $a$ is a Ramanujan sum, adding up to -1 . If $\chi_{2} \chi_{4} \chi_{8}$ is non-principal, then the substitution $b=-a m$ transforms the sum over $a$ into the Gauß sum $\tau\left(\chi_{2} \chi_{4} \chi_{8}\right)$, save for a factor of modulus 1 . This yields $|A(p, m)| \leq 21 p^{-1}$, as required.

Lemma 8. Let $m \in \mathbb{Z}$, and let $p$ be an odd prime. Then $A\left(p^{2}, m\right)=p^{-3} c_{p^{2}}(m)$.

Proof. This is immediate from 4.6 , 5.2 and $(5.3)$.

LEMma 9. Let $m \in \mathbb{Z}$, let $p$ be an odd prime and suppose that $3 \leq u \leq 8$. If $p^{u-1} \nmid m$, then $A\left(p^{u}, m\right)=0$. If $p^{u-1} \mid m$ and $u$ is even, then

$$
A\left(p^{u}, m\right) \ll p^{-1}\left(p, m p^{1-u}\right) \quad(u=4,6), \quad A\left(p^{8}, m\right) \ll\left(p, m / p^{7}\right) .
$$

If $p^{u-1} \mid m$ and $u$ is odd, then $A\left(p^{u}, m\right) \ll p^{-1 / 2}\left(p, m p^{1-u}\right)^{1 / 2}$. One has $A\left(p^{3}, m\right) \ll p^{-1}$, and if $p^{3} \mid m$ then $A\left(p^{3}, m\right)=0$. Finally, one has $A\left(p^{7}, m\right) \ll 1$, and if $p^{7} \mid m$ then $A\left(p^{7}, m\right)=0$. 
Proof. In the case where $u$ is even, we apply (4.6), (5.2) and $(5.3)$ and infer

$$
A\left(p^{u}, m\right)=p^{-u} c_{p^{u}}(m) \quad(u=4,6), \quad A\left(p^{8}, m\right)=p^{-7} c_{p^{8}}(m) .
$$

All claims now follow from the evaluation of Ramanujan's sum.

Now let $u=7$. By $(5.2),(5.3)$ and $(5.5)$, we find that

$$
A\left(p^{7}, m\right)=\frac{S_{2}(p, 1)}{p^{7}} \sum_{\substack{a=1 \\ p \nmid a}}^{p^{7}}\left(\frac{a}{p}\right) e\left(-\frac{a m}{p^{7}}\right)=\frac{S_{2}(p, 1)}{p^{7}} \sum_{b=1}^{p-1} \sum_{c=1}^{p^{6}}\left(\frac{b}{p}\right) e\left(-\frac{(b+p c) m}{p^{7}}\right) .
$$

The sum over $c$ vanishes except when $p^{6} \mid m$, and in that case, we write $m=p^{6} m^{\prime}$ to infer

$$
A\left(p^{7}, m\right)=\frac{S_{2}(p, 1)}{p} \sum_{b=1}^{p-1}\left(\frac{b}{p}\right) e\left(-\frac{b m^{\prime}}{p}\right) .
$$

If $p \mid m^{\prime}$ then the sum over $b$ vanishes, and if $p \nmid m^{\prime}$, then we may sum over $b m^{\prime}$ in place of $b$ to see that the sum over $b$ is a quadratic Gauss sum. This establishes all claims concerning the case $u=7$.

The case $u=3$ is very similar: here $5.2,5$, 5.3 and 5.5 give

$$
A\left(p^{3}, m\right)=\frac{S_{2}(p, 1)}{p^{4}} \sum_{\substack{a=1 \\ p \nmid a}}^{p^{3}}\left(\frac{a}{p}\right) e\left(-\frac{a m}{p^{3}}\right) .
$$

Proceeding as before, we find that the sum on the right vanishes unless $p^{2} \mid m$, and in that case we write $m=p^{2} m^{\prime}$ to conclude that

$$
A\left(p^{3}, m\right)=\frac{S_{2}(p, 1)}{p^{2}} \sum_{b=1}^{p-1}\left(\frac{b}{p}\right) e\left(-\frac{b m^{\prime}}{p}\right) .
$$

We now argue as in the case $u=7$ to confirm all claims concerning the case $u=3$.

This leaves the case $u=5$. By 5.2 and (5.3),

$$
\begin{aligned}
A\left(p^{5}, m\right)=\frac{1}{p^{6}} \sum_{\substack{a=1 \\
p \nmid a}}^{p^{5}} S_{2}(p, a) S_{4}(p, a) e(- & \left.\frac{a m}{p^{5}}\right) \\
& =\frac{1}{p^{6}} \sum_{b=1}^{p-1} \sum_{c=1}^{p^{4}} S_{2}(p, b) S_{4}(p, b) e\left(-\frac{(b+p c) m}{p^{5}}\right) .
\end{aligned}
$$

As before, the sum over $c$ vanishes unless $p^{4} \mid m$. In that case, we write $m=p^{4} m^{\prime}$, and then find via $(5.4)$ and $(5.5)$ that

$$
A\left(p^{5}, m\right)=\frac{S_{2}(p, 1)}{p^{2}} \sum_{\chi_{4} \in \mathrm{X}_{4}} \tau\left(\bar{\chi}_{4}\right) \sum_{b=1}^{p-1}\left(\frac{b}{p}\right) \chi_{4}(b) e\left(-\frac{b m^{\prime}}{p}\right) .
$$

The Legendre symbol is among the characters in $\mathrm{X}_{4}$, and for this character the sum over $b$ is $c_{p}\left(m^{\prime}\right)$. Hence, the Legendre symbol contributes to $A\left(p^{5}, m\right)$ an amount bounded by $p^{-1}\left(p, m^{\prime}\right)$ in modulus. If the Legendre symbol is the only character in $\mathrm{X}_{4}$ then we are finished. Otherwise, there are two further characters in $\mathrm{X}_{4}$, both of order 4 . When multiplied with the Legendre symbol, they remain non-principal, so that the sum over $b$ 
vanishes when $p \mid m^{\prime}$, but transforms into a Gauss sum when $p \nmid m^{\prime}$. This confirms all claims concerning the case $u=5$.

The following rough version of our earlier lemmata is readier to use, and often suffices.

Lemma 10. Let $m \in \mathbb{Z}$, and let $p$ be an odd prime. Suppose that $t \geq 2$. Then $A\left(p^{t}, m\right)=0$ unless $p^{t-1} \mid m$ in which case one has $A\left(p^{t}, m\right) \ll p^{[t / 8]-1 / 2}\left(p, m p^{1-t}\right)^{1 / 2}$.

Proof. Write $t=u+8 v$ with $1 \leq u \leq 8$. Suppose that $A\left(p^{t}, m\right) \neq 0$. Repeated use of Lemma 6 shows that $p^{8 v} \mid m$, and that $A\left(p^{t}, m\right)=p^{v} A\left(p^{u}, m p^{-8 v}\right)$. Note here that $[t / 8]=v$ for $u \leq 7$, and $[t / 8]=v+1$ for $u=8$. If $u=1$, then the lemma follows from Lemma 7. If $u=2$ then it can be deduced from Lemma 8, and if $u \geq 3$, then from Lemma 9

We require similar information for $p=2$ but do not need to be so precise. Hence, we suppose that $t \geq 10$, and write $t=8 v+u$ with $2 \leq u \leq 9$. Then, by Lemma 6, we have $A\left(2^{t}, m\right)=0$ unless $2^{8 v} \mid m$ in which case

$$
A\left(2^{t}, m\right)=2^{v} A\left(2^{u}, 2^{-8 v} m\right) .
$$

Finally we study the value of $A\left(p^{t}, n-y^{6}\right)$ as $y$ varies.

LEMMA 11. Let $t \geq 2$, let $p$ be an odd prime dividing $n$, and let $p^{\nu} \| n$.

(i) Let $t \leq \nu+1$. If $6 \nmid t-1$ then $A\left(p^{t}, n-y^{6}\right)=0$, except when $p^{t} \mid y^{6}$, and in that case $A\left(p^{t}, n-y^{6}\right)=A\left(p^{t}, n\right)$. If $6 \mid t-1$ then $A\left(p^{t}, n-y^{6}\right)=0$, except when $p^{(t-1) / 6} \mid y$.

(ii) Let $t \geq \nu+2$. Then $A\left(p^{t}, n-y^{6}\right)=0$, except when $6 \mid \nu$ and $p^{\nu / 6} \mid y$.

Proof. Put $n=p^{\nu} n^{\prime}$. By Lemma 10 we see that $A\left(p^{t}, n-y^{6}\right) \neq 0$ implies $p^{t-1} \mid p^{\nu} n^{\prime}-y^{6}$.

First suppose that $t \leq \nu+1$. Then, it follows that $p^{t-1} \mid y^{6}$, and if $6 \nmid t-1$, we may conclude that $p^{t} \mid y^{6}$. For $t \leq \nu$, we now see that $p^{t} \mid n-y^{6}$, and hence, $A\left(p^{t}, n-y^{6}\right)=$ $A\left(p^{t}, p^{t}\right)=A\left(p^{t}, n\right)$. For $t=\nu+1$ and $6 \nmid t-1=\nu$, we still have $p^{t} \mid y^{6}$ and $A\left(p^{t}, n-y^{6}\right)=$ $A\left(p^{t}, n\right)$. This verifies the first clause of (i).

Now consider the case $6 \mid t-1$. Then $p^{t-1} \mid y^{6}$ is the same as $p^{(t-1) / 6} \mid y$. This establishes (i).

Finally, suppose that $t \geq \nu+2$. Then $p^{t-1} \mid p^{\nu} n^{\prime}-y^{6}$ is impossible unless $6 \mid \nu$ and $p^{\nu / 6} \mid y$. This is (ii).

We now turn to the sum $B\left(p^{t}, n\right)$. In the discussion, it will be convenient to have at hand an estimate for the number $\psi_{n}(q)$ of incongruent solutions to $y^{6} \equiv n \bmod q$.

LEMma 12. For each $n \in \mathbb{N}$ the function $\psi_{n}$ is multiplicative. Let $p$ is an odd prime with $n=p^{\nu} n^{\prime}$ and $p \nmid n^{\prime}$. Then, for $1 \leq t \leq \nu$ one has $\psi_{n}\left(p^{t}\right)=p^{t-1-[(t-1) / 6]}$. For $t>\nu$ one has $\psi_{n}\left(p^{t}\right)=0$ except when $6 \mid \nu$ where $\psi_{n}\left(p^{t}\right) \leq 6 p^{5 \nu / 6}$.

This belongs to the elementary theory of congruences, and needs no proof here. It should be noted, however, that we have not excluded the case $\nu=0$.

Lemma 13. Let $t=1$ or 2 . Then, uniformly in $n \in \mathbb{N}$ and primes $p$, one has $B\left(p^{t}, n\right) \ll 1$.

Proof. We may suppose that $p>2$ since the claim is trivial for $p=2$. Since there are no more than 6 incongruent solutions to $b^{6} \equiv n \bmod p$, the bound for $B(p, n)$ follows 
immediately from 5.1 and Lemma 7 Further, by Lemma 8

$$
B\left(p^{2}, n\right)=p^{-3} \sum_{b=1}^{p^{2}}\left|c_{p^{2}}\left(n-b^{6}\right)\right|
$$

The evaluation of Ramanujan's sum shows that only those $b$ where $b^{6} \equiv n \bmod p$ make a non-zero contribution, and there are no more than $6 p$ such $b$ with $1 \leq b \leq p^{2}$. The trivial bound $\left|c_{p^{2}}(m)\right| \leq p^{2}$ now yields $B\left(p^{2}, n\right) \leq 6$, as required.

For larger powers of an odd prime $p$, we begin with a crude bound that is uniform in $n$. From (5.1) and Lemma 10 we see that for $t \geq 3$ one has

$$
B\left(p^{t}, n\right) \leq\left(\psi_{n}\left(p^{t-1}\right) p^{1 / 2}+\psi_{n}\left(p^{t}\right)\right) p^{[t / 8]} .
$$

Now $t>\nu$ implies $t-1-[(t-1) / 6] \geq 5 \nu / 6$. By Lemma 11] $B\left(p^{t}, n\right) \leq 12 p^{M}$ where

$$
M=\left[\frac{t}{8}\right]+\max \left(t-\frac{3}{2}-\left[\frac{t-2}{6}\right] ; t-1-\left[\frac{t-1}{6}\right]\right) .
$$

It follows that $M \leq t-1$. Further, $\left[\frac{t-1}{6}\right] \geq\left[\frac{t}{8}\right]+1$ holds for all $t \geq 25$, so that one then has $M \leq t-\frac{3}{2}$. Finally, the trivial inequalities $\left[\frac{t-2}{6}\right] \geq \frac{t-2}{6}-\frac{5}{6}$ and $\left[\frac{t-1}{6}\right] \geq \frac{t-1}{6}-\frac{5}{6}$ imply $M \leq \frac{23}{24} t$. We summarise these findings.

LEMMA 14. Uniformly in $n$ and primes $p$, one has

$$
B\left(p^{t}, n\right) \ll p^{t-1}(t \geq 3), \quad B\left(p^{t}, n\right) \ll p^{t-3 / 2}(t \geq 25), \quad B\left(p^{t}, n\right) \ll p^{23 t / 24} .
$$

If $p^{\nu} \| n$ and $0 \leq \nu \leq 4$ then

$$
B\left(p^{t}, n\right) \ll p^{t-3 / 2}
$$

holds for all $t \geq 3$. If $\nu=6$ then (5.8) holds for $t \geq 9$, if $\nu=12$ then (5.8) holds for $t \geq 13$, and if $\nu=18$ then for $t \geq 19$.

Proof. We begin by considering odd primes $p$. In this case the first clause has already been proved, so we may concentrate on (5.8). If $\nu=0$ then Lemma 12 gives $\psi_{n}\left(p^{l}\right) \leq 6$ for all $l \geq 1$, and so, by (5.7) we have $B\left(p^{t}, n\right) \ll p^{1 / 2+[t / 8]}$ which is much better than claimed. For $\nu=1$ Lemma 12 yields $\psi_{n}\left(p^{l}\right)=0$ for $l \geq 2$, and (5.7) gives $B\left(p^{t}, n\right)=0$ for $t \geq 3$. The same argument applies for $\nu=2, t \geq 4$ and $\nu=3, t \geq 5$. By Lemma 9 .

$$
B\left(p^{3}, p^{2} n^{\prime}\right)=\sum_{\substack{b=1 \\ b^{6} \equiv p^{2} n^{\prime} \bmod p^{2}}}^{p^{3}}\left|A\left(p^{3}, p^{2} n^{\prime}-b^{6}\right)\right|=p^{2}\left|A\left(p^{3}, p^{2} n^{\prime}\right)\right| \ll p,
$$

while a similar computation delivers

$$
B\left(p^{3}, p^{3} n^{\prime}\right)=p^{2}\left|A\left(p^{3}, p^{3}\right)\right|=0, \quad B\left(p^{4}, p^{3} n^{\prime}\right)=\sum_{\substack{b=1 \\ b^{6} \equiv p^{3} n^{\prime} \bmod p^{3}}}^{p^{4}}\left|A\left(p^{4}, p^{3} n^{\prime}\right)\right| \ll p^{2} .
$$

This proves 5.8 for $\nu \leq 4$. The cases of 5.8 where $\nu=6,12$ or 18 all follow from (5.7) coupled with the final clause of Lemma 12 The proof is complete for odd primes. 
Now consider $p=2$. Let $t \geq 25$ and write $t=8 v+u$ with $2 \leq u \leq 9$, as in 5.6, which gives

$$
B\left(2^{t}, n\right)=\sum_{b=1}^{2^{t}}\left|A\left(2^{t}, n-b^{6}\right)\right|=2^{v} \sum_{\substack{b=1 \\ 2^{8 v} \mid n-b^{6}}}^{2^{t}}\left|A\left(2^{u}, 2^{-8 v}\left(n-b^{6}\right)\right)\right| .
$$

Elementary counting of the solutions of $n-b^{6} \equiv 0 \bmod 2^{8 v}$ now shows that the bound $B\left(p^{t}, n\right) \ll p^{23 t / 24}$ also holds for $p=2$, at least for $t \geq 25$. For $t \leq 25$, the sum $B\left(2^{t}, n\right)$ takes only finitely many values, and therefore, all other claims in the lemma are true and trivial for $p=2$.

6. Higher powered tristesse. Equipped with sufficient information about the function $A(q, m)$ and its relatives, we now return to our main theme. In view of Lemma 4, the next task ahead of us is the asymptotic evaluation of $\sigma(n, N)$, as defined in (4.14). This sum involves the smooth monotonic weight $\left(n-y^{6}\right)^{-1 / 8}$ that can be removed by partial summation. Thus, we are led to study the sum

$$
\Upsilon_{n}(P)=\Upsilon_{n}(P, Q)=\sum_{y \in \mathcal{A}\left(P, P_{6}^{\eta}\right)} \sum_{q \leq Q} A\left(q, n-y^{6}\right)
$$

where we will always choose $Q=N^{1 / 13}$ and assume that

$$
\frac{1}{2} N<n \leq N, \quad 8 \nmid n, \quad P_{6} \exp (-\sqrt{ } \log N) \leq P \leq \frac{1}{2} P_{6},
$$

smaller $P$ being of lesser relevance.

In this and the next two sections we establish an asymptotic formula for $\Upsilon_{n}(P)$. The basic idea is one of great simplicity. Since the summand $A\left(q, n-y^{6}\right)$ depends only on $y \bmod q$, one sorts the smooth $y$ into progressions, modulo $q$, and then uses results on their distribution in these progressions on average over $q$. Unfortunately $A\left(q, n-y^{6}\right)$ is sometimes too large for a straightforward treatment. It turns out that higher powers of primes dividing $q$ do not cooperate with an estimate of Bombieri-Vinogradov type, in particular if these prime powers also divide $n$. We therefore initiate the analysis with a chain of preparatory estimates to mollify the effects of undesired factors.

For natural numbers $q$ and $k \geq 2$ let

$$
q_{k}=\prod_{\substack{p^{l} \| q \\ l \geq k}} p^{l}
$$

denote the $k$-full part of $q$. Throughout this section, but this section only, an index attached to a natural number will always have this meaning. We begin by examining the contribution to 6.1 arising from $q$ where $q_{25}$ is large. Let $L \geq 1$ be a parameter. Then, by Rankin's trick followed by sorting the $y$-summation in arithmetic progressions, we find

$$
\begin{aligned}
\sum_{\substack{q \leq Q \\
q 25}} \sum_{y \in \mathcal{A}\left(P, P_{6}^{\eta}\right)}\left|A\left(q, n-y^{6}\right)\right| & \leq \sum_{q \leq Q}\left(\frac{q_{25}}{L}\right)^{1 / 96} \sum_{b=1}^{q}\left|A\left(q, n-b^{6}\right)\right| \sum_{\substack{y \leq P \\
y \equiv b \bmod q}} 1 \\
& \ll P L^{-1 / 96} \sum_{q \leq Q} q^{-1} q_{25}^{1 / 96} B(q, n) .
\end{aligned}
$$


Note that we have used here that $q \leq Q \leq P$. By Lemma 14, there is a number $C \geq 1$ with $B\left(p^{t}, n\right) \leq C p^{t-1}$ for all primes $p$, all $t \geq 1$. Further, again by Lemma 14, when $t \geq 25$, one has

$$
p^{-95 t / 96} B\left(p^{t}, n\right) \ll \min \left(p^{-3 / 2+t / 96}, p^{-t / 32}\right),
$$

and so, by multiplicativity of $B$, one finds that the right hand side of $(6.3)$ is bounded by

$$
\begin{aligned}
& \ll P L^{-1 / 96} \prod_{p \leq Q}\left(1+\sum_{t=1}^{24} p^{-t} B\left(p^{t}, n\right)+\sum_{t=25}^{\infty} p^{-95 t / 96} B\left(p^{t}, n\right)\right) \\
& \ll P L^{-1 / 96} \prod_{p \leq Q}\left(1+\frac{24 C}{p}+O\left(p^{-33 / 32}\right)\right) \ll P L^{-1 / 96}(\log N)^{24 C} .
\end{aligned}
$$

As the simplest application, we take $L=1$ and then have

$$
\sum_{q \leq Q} \sum_{y \in \mathcal{A}\left(P, P_{6}^{\eta}\right)}\left|A\left(q, n-y^{6}\right)\right| \ll P(\log N)^{24 C} .
$$

Similarly, we may take

$$
L=(\log N)^{2400 C}
$$

and then infer that

$$
\sum_{\substack{q \leq Q \\ q_{25}>L}} \sum_{y \in \mathcal{A}\left(P, P_{6}^{\eta}\right)}\left|A\left(q, n-y^{6}\right)\right| \ll P(\log N)^{-C} .
$$

Note that we were able to control $q_{25}$ because the estimate $p^{-t} B\left(p^{t}, n\right) \ll p^{-3 / 2}$ was available for $t \geq 25$. However, when $p \nmid n_{4}$ then Lemma 14 provides this critical estimate already for $t \geq 3$. Therefore, we define

$$
\mathcal{Q}_{n}=\left\{q \in \mathbb{N}: p\left|q \Rightarrow p^{4}\right| n\right\} .
$$

Then, any natural number $q$ factorises uniquely into $q=q^{*} q^{\dagger}$ with $q^{\dagger} \in \mathcal{Q}_{n}$ and $\left(q^{*}, n_{4}\right)=1$. Although this factorisation depends on $n$, we have suppressed this in the notation because we now work with only one large $n$. In accordance with the conventions introduced earlier in this section, the cubefull part of $q^{*}$ is $q_{3}^{*}$, for example. We may now copy the argument from $(6.3)$ to 6.7 to confirm the estimate

$$
\sum_{\substack{q \leq Q \\ q_{3}^{*}>L}} \sum_{\substack{\text { A } \\\left(P, P_{6}^{\eta}\right)}}\left|A\left(q, n-y^{6}\right)\right| \ll P(\log N)^{-C},
$$

where from now on $L$ is always given by 6.6 . To verify 6.8 , one applies Rankin's trick with $\left(q_{3}^{*} / L\right)^{1 / 96}$. The analogue of 6.4 then becomes

$$
P L^{-1 / 96} \prod_{\substack{p \leq Q \\ p \mid n_{4}}}\left(1+\frac{24 C}{p}+O\left(p^{-33 / 32}\right)\right) \prod_{\substack{p \leq Q \\ p \nmid n_{4}}}\left(1+\frac{2 C}{p}+\sum_{t=3}^{\infty} p^{-95 t / 96} B\left(p^{t}, n\right)\right),
$$

and from here, the estimation is completed as before. Further, we may factor $q^{\dagger}$ as

$$
q^{\dagger}=q^{(4)} q^{(5)} q^{(6)} \ldots
$$


where $q^{(\nu)}$ is composed of primes $p$ with $p^{\nu} \| n$ only. Again, this factorisation is unique, and we may use Lemma 14 again to control the size of $q_{9}^{(6)}, q_{13}^{(12)}$ and $q_{19}^{(18)}$, where again we find that the portion of (6.5) where at least one of these three numbers exceeds $L$, is bounded by $\left.O\left(P(\log N)^{-C}\right)\right)$.

Finally we limit the size of $q_{3}^{\dagger}$. Unfortunately we are unable to do this on the logarithmic scale. Thus, put

$$
K=\exp (\sqrt{ } \log N),
$$

and follow the ideas in 6.3 to see that

$$
\begin{aligned}
\sum_{\substack{q \leq Q \\
q_{3}^{\dagger}>K}}\left|A\left(q, n-y^{6}\right)\right| & \ll P K^{-1 / 96} \prod_{\substack{p \leq Q \\
p \nmid n_{4}}}\left(1+\frac{24 C}{p}\right) \prod_{p \mid n_{4}}\left(1+C \sum_{t=1}^{24} p^{t / 96-1}+O\left(p^{-33 / 32}\right)\right) \\
& \ll P K^{-1 / 97} \prod_{p^{4} \mid n}\left(1+24 C p^{-3 / 4}\right) \ll P K^{-1 / 98} .
\end{aligned}
$$

We summarise our findings. Let $L, K$ be given by 6.6, 6.10). Then define $\Upsilon_{n}^{\prime}(P)$ to denote the portion of the sum (6.1) where $q$ is restricted by the extra conditions

$$
q_{25} \leq L, \quad q_{3}^{*} \leq L, \quad q_{3}^{\dagger} \leq K, \quad q_{9}^{(6)} \leq L, \quad q_{13}^{(12)} \leq L, \quad q_{19}^{(18)} \leq L .
$$

On combining (6.7), 6.8), 6.11) and the discussion following 6.9), we deduce that

$$
\Upsilon_{n}(P)=\Upsilon_{n}^{\prime}(P)+O\left(P(\log N)^{-1}\right)
$$

7. Trouble ahead. We now embark on the approximation argument that ultimately removes the smoothness condition from the $y$-sum in 6.1). This is a technically very demanding endeavour. Most of the difficulties arise from prime divisors of the 4-full part of $n$, and if one were prepared to consider 4 -free $n$ only then a much more economic treatment would be possible.

The starting point is the restricted sum $\Upsilon_{n}^{\prime}(P)$, as defined via (6.12). We present this sum in a form more suitable for the argument to follow. Let $\mathcal{U}$ denote the set of cubefree numbers, and let

$$
\mathcal{V}=\left\{v \in \mathbb{N}: p\left|v \Rightarrow p^{3}\right| v, p^{4} \mid n\right\}, \quad \mathcal{W}=\left\{w \in \mathbb{N}: p\left|w \Rightarrow p^{3}\right| w, p^{4} \nmid n\right\} .
$$

Then, a number $q \in \mathbb{N}$ factors uniquely as $q=u v w$ with $u \in \mathcal{U}, v \in \mathcal{V}, w \in \mathcal{W}$ pairwise coprime. Note that $w=q_{3}^{*}, v=q_{3}^{\dagger}$ in the notation of the previous section that we do no longer use now. Recall also $8 \nmid n$ so that all $v \in \mathcal{V}$ are odd. We factor $v$ further. Let $p$ be a prime with $p^{t} \in \mathcal{V}$ and $p^{\nu} \| n$. We say that $p^{t}$ is of

- class 1 if $t \not \equiv 1 \bmod 6,3 \leq t \leq \min (\nu+1,24)$,

- class 2 if $t \equiv 1 \bmod 6,3 \leq t \leq \min (\nu+1,24)$ except when $\nu=12$ and 18 , in which case $3 \leq t \leq \min (\nu, 24)$,

- class 3 if $t=8, \nu=6$,

- class 4 if $p^{t}$ is in none of the classes 1,2 and 3.

Let $\mathcal{V}_{j}$ be the set of all $v \in \mathcal{V}$ composed of $p^{t} \| v$ with $p^{t}$ of class $j$ only. Then, $v$ factors uniquely into $v=v_{1} v_{2} v_{3} v_{4}$ with $v_{j} \in \mathcal{V}_{j}$ coprime in pairs, and a given $q$ factors as $q=u v w=u v_{1} v_{2} v_{3} v_{4} w$. We use this factorisation throughout this section. 
We recast the conditions 6.12 in terms of this factorisation. Given $q$, first consider the corresponding $v_{4}=v_{4}(q) \in \mathcal{V}_{4}$. Let $p^{t} \| v_{4}$ and $p^{\nu} \| n$. If $t \geq 25$ then $p^{t}$ is a divisor of $q_{25}$ in 6.12 , and hence of $q_{25}^{\dagger}$. Next suppose that $\nu+2 \leq t \leq 24,6 \nmid \nu$. Then, by Lemma 11. $A\left(p^{t}, n-y^{6}\right)=0$ for all $y$, and hence, $A\left(q, n-y^{6}\right)=0$ for all $y$; such $q$ may be omitted from the sum defining $\Upsilon_{n}^{\prime}(P)$. If $\nu+2 \leq t \leq 24$ and $6 \mid \nu$, then $\nu=6,12$ or 18 , and $p^{t} \mid q_{9}^{(6)} q_{13}^{(12)} q_{19}^{(18)}$. This shows that $v_{4} \mid q_{25}^{\dagger} q_{9}^{(6)} q_{13}^{(12)} q_{19}^{(18)}$, in the notation of 6.12 , and we then see that $v_{4}$ actually equals $q_{25}^{\dagger} q_{9}^{(6)} q_{13}^{(12)} q_{19}^{(18)}$. In particular, the definition of class 4 could also be given directly, in terms of $\nu$ and $t$. Further, we see that any $q \in \mathbb{N}$ with $q \leq Q$ that satisfies 6.12 and is such that $A\left(q, n-y^{6}\right)$ does not vanish identically in $y$, has exactly one factorisation $q=u v_{1} v_{2} v_{3} v_{4} w$ as above, with $u \in \mathcal{U}, v_{j} \in \mathcal{V}_{j}, w \in \mathcal{W}$ and

$w \leq L, v_{1} v_{2} v_{3} v_{4} \leq K, v_{4} \leq L^{4}, u v w \leq Q, u, v_{1}, v_{2}, v_{3}, v_{4}, w$ coprime in pairs.

Conversely, if $u, v_{j}$ and $w$ run independently over these conditions, with $v_{4}$ restricted to a suitable subset, we cover all $q$ satisfying 6.12 .

Next, we extract more consequences of Lemma 11. Let $q \in \mathbb{N}$ and $n \in \mathbb{N}$ be given. We say that the number $s$ is a significant divisor of $q$ (relative to $n$ ) if $s \mid q$ and for all $y \in \mathbb{N}$ with $A\left(q, n-y^{6}\right) \neq 0$ one has $s \mid y$. Note that 1 is always a significant divisor. Also, there is a multiplicative property: if $q_{1}$ and $q_{2}$ are coprime with significant divisors $s_{j}$ of $q_{j}$, then $s_{1} s_{2}$ is a significant divisor of $q_{1} q_{2}$. To see this, note that Lemma 5 shows that $A\left(q_{1} q_{2}, n-y^{6}\right) \neq 0$ implies $A\left(q_{j}, n-y^{6}\right) \neq 0$, and then we have $s_{j} \mid y$ for $j=1,2$, which in turn gives $s_{1} s_{2} \mid y$, as required.

We now determine significant divisors of $p^{t} \in \mathcal{V}$ in classes 1,2 and 3. For $p^{t}$ of class 1 , Lemma 11 tells us that $s\left(p^{t}\right)=p^{1+[(t-1) / 6]}$ is a significant divisor, and for class 2 we may take $s\left(p^{t}\right)=p^{(t-1) / 6}$. For $p^{t}$ of class 3 , we have $t=8$ and $\nu=6$, and again by Lemma 11 . $s\left(p^{8}\right)=p$ is a significant divisor. Now, if $p^{t}$ is a prime power of class 4 , or a prime power not in $\mathcal{V}$, then we take $s\left(p^{t}\right)=1$ and extend $s$ to a multiplicative function on $\mathbb{N}$. Then $s(q)$ is a significant divisor of $q$, for all $q \in \mathbb{N}$, and if $q$ is translated into the form 7.1), then $s(q)=s(v)=s\left(v_{1}\right) s\left(v_{2}\right) s\left(v_{3}\right)$. By (7.1) and the definition of $\Upsilon_{n}^{\prime}(P)$ in 6.12 , we get

$$
\begin{aligned}
\Upsilon_{n}^{\prime}(P)=\sum_{\substack{q<Q \\
6.12}} \sum_{z \in \mathcal{A}\left(P / s(q), P_{6}^{\eta}\right)} A\left(q, n-s(q)^{6} z^{6}\right) \\
\quad=\sum_{\substack{u, v, w \\
7.1}} \sum_{z \in \mathcal{A}\left(P / s(v), P_{6}^{\eta}\right)} A\left(q, n-s(v)^{6} z^{6}\right) .
\end{aligned}
$$

The reader may have expected the condition $s(q) \in \mathcal{A}\left(Q, P_{6}^{\eta}\right)$ in the outer sums, but $s(q)=s(v)$ divides $v_{1} v_{2} v_{3}$, and in view of (7.1) we have $s(q) \leq K \leq P_{6}^{\eta}$, so the smoothness is automatically guaranteed. In 7.2 , we factor $A(u v w, m)$, and begin with the divisor $v_{1}$. We take $y=s\left(v_{2} v_{3}\right) z$ and apply Lemma 11 and multiplicativity to see that

$$
A\left(v_{1}, n-s(v)^{6} z^{6}\right)=A\left(v_{1}, n-s\left(v_{1}\right)^{6} y^{6}\right)=A\left(v_{1}, n\right)
$$

is independent of $z$. Further, from the definition of $s$ we find that $s\left(v_{2}\right)^{6} \mid v_{2}$, and that $s\left(v_{3}\right)^{8}=v_{3}$. In particular, the factor $A\left(v_{2} v_{3}, n-s(v)^{6} z^{6}\right)$ depends only on $z$ modulo $v_{2} v_{3} / s\left(v_{2} v_{3}\right)^{6}$. For notational simplicity, we now put

$$
r=u w v_{2} v_{3} v_{4} / s\left(v_{2} v_{3}\right)^{6}
$$


and then infer that

$$
\Upsilon_{n}^{\prime}(P)=\sum_{v_{1} \in \mathcal{V}_{1}} A\left(v_{1}, n\right) \sum_{\substack{u, v_{2}, v_{3}, v_{4}, w \\ 7.1}} \sum_{b=1}^{r} A\left(u v_{2} v_{3} v_{4} w, n-s(v)^{6} b^{6}\right) \Psi\left(P / s(v), P_{6}^{\eta}, r, b\right)
$$

where $\Psi(X, Y, q, a)$ denotes the number of $m \in \mathcal{A}(X, Y)$ with $m \equiv a$ mod $q$. Similarly, we denote the number of $m \in \mathcal{A}(X, Y)$ with $(m, q)=1$ by $\Psi_{q}(X, Y)$, and put $\Psi(X, Y)=\Psi_{1}(X, Y)$. Then define

$$
D_{q}^{*}(X, Y)=\max _{(a, q)=1}\left|\Psi(X, Y, q, a)-\frac{\Psi_{q}(X, Y)}{\varphi(q)}\right|, \quad D_{q}(Z, Y)=\max _{1 \leq X \leq Z} D_{q}^{*}(X, Y) .
$$

Lemma 15. Fix real numbers $\delta>0, A \geq 1$ and $C \geq 1$. Then, whenever $1 \leq Q \leq Z^{1 / 2-\delta}$ one has

$$
\sum_{q \leq Q} C^{\omega(q)} D_{q}(Z, Y) \ll Z(\log Z)^{-A}
$$

Proof. This is a rough version of a Bombieri-Vinogradov theorem for smooth numbers, and the case $C=1$ is due to Wolke [16]. For larger values of $C$, we apply the case $C=1$ with $2 A$ in place of $A$. Then, for $C>1$, the contribution of those $q$ where $C^{\omega(q)} \leq(\log Z)^{A}$ is bounded by $O\left(Z(\log Z)^{-A}\right)$, as desired. To estimate the sum over $q$ with $C^{\omega(q)}>(\log Z)^{A}$, we use Rankin's trick and the trivial bound $D(q, Z) \ll Z / \varphi(q)$. Then this part of the sum is

$$
\ll(\log Z)^{-2 A} \sum_{q \leq Q} \frac{C^{3 \omega(q)}}{\varphi(q)} \ll(\log Z)^{C^{3}-2 A},
$$

and the result follows, at least for large $A$, and this suffices.

We wish to use this within (7.5), and therefore arrange the sum over $b$ according to $d=(b, r)$. In that situation, any $m \equiv b \bmod r$ counted by $\Psi\left(P / s(v), P_{6}^{\eta}, r, b\right)$ will have $d \mid m$, and this is possible only when $d \in \mathcal{A}\left(r, P_{6}^{\eta}\right)$. From now on, the smoothness parameter ( $Y$ in Lemma 15 in our discussion below will always be $P_{6}^{\eta}$, and for brevity we drop this from the notation. Thus, we now write $\mathcal{A}(r)$ for $\mathcal{A}\left(r, P_{6}^{\eta}\right)$ and $\Psi(X, r, b)$ for $\Psi\left(X, P_{6}^{\eta}, r, b\right)$, for example. Also, we put $s=s(v)$ and $s_{j}=s\left(v_{j}\right)$ whenever there is need to save space. In this notation, by (7.5), the sum $\Upsilon_{n}^{\prime}(P)$ equals

$$
\begin{aligned}
& =\sum_{v_{1} \in \mathcal{V}_{1}} A\left(v_{1}, n\right) \sum_{\substack{u, v_{2}, v_{3}, v_{4}, w \\
[7.1}} \sum_{\substack{d \mid r \\
d \in \mathcal{A}(r)}} \sum_{\substack{b=1 \\
(b, r)=d}}^{r} A\left(u v_{2} v_{3} v_{4} w, n-s^{6} b^{6}\right) \Psi\left(\frac{P}{d s}, \frac{r}{d}, \frac{b}{d}\right) \\
& =\sum_{v_{1} \in \mathcal{V}_{1}} A\left(v_{1}, n\right) \sum_{\substack{\left.u, v_{2}, v_{3}, v_{4}, w \\
7.1\right]}} \sum_{\substack{d \mid r \\
d \in \mathcal{A}(r)}} \sum_{\substack{\left(b^{\prime}, r / d\right)=1 \\
b^{\prime}=1}}^{r / d} A\left(u v_{2} v_{3} v_{4} w, n-s^{6} d^{6} b^{\prime 6}\right) \Psi\left(\frac{P}{d s}, \frac{r}{d}, b^{\prime}\right),
\end{aligned}
$$

and we replace $\Psi$ with $\Psi_{r / d}$ to arrive at

$$
\Upsilon_{n}^{\prime}(P)=\mathrm{M}_{n}(P)+\mathrm{E}_{n}(P)
$$


where $\mathrm{M}_{n}(P)$ is the sum

$$
\sum_{v_{1} \in \mathcal{V}_{1}} A\left(v_{1}, n\right) \sum_{\substack{u, v_{2}, v_{3}, v_{4}, w \\[7.1]}} \sum_{\substack{d \mid r \\ d \in \mathcal{A}(r)}} \sum_{\substack{b=1 \\ b=r / d)=1}}^{r / d} A\left(u v_{2} v_{3} v_{4} w, n-s^{6} d^{6} b^{6}\right) \varphi\left(\frac{r}{d}\right)^{-1} \Psi_{r / d}\left(\frac{P}{d s}\right),
$$

and, in view of 7.6 , we have

$$
\begin{aligned}
& \left|\mathrm{E}_{n}(P)\right| \\
& \leq \sum_{v_{1} \in \mathcal{V}_{1}}\left|A\left(v_{1}, n\right)\right| \sum_{\substack{u, v_{2}, v_{3}, v_{4}, w \\
(7.1)}} \sum_{d \mid r} \sum_{\substack{b=1 \\
(b, r / d)=1}}^{r / d}\left|A\left(u v_{2} v_{3} v_{4} w, n-s^{6} d^{6} b^{6}\right)\right| D_{r / d}^{*}\left(\frac{P}{d s}\right) .
\end{aligned}
$$

Note that in 7.9 we dropped the smoothness of $d$ to produce an upper bound. We now concentrate on the sum over $b$. We substitute $s\left(v_{1}\right) b$ for $b$, observing that $\left(s\left(v_{1}\right), r\right)=1$, and then see that we may replace the factor $s^{6}=s(v)^{6}$ with $s\left(v_{2} v_{3}\right)^{6}$. Next suppose that $u v_{2} v_{3} v_{4} w=q^{\prime} q^{\prime \prime}$ with $\left(q^{\prime}, q^{\prime \prime}\right)=1$. Then $s\left(v_{2} v_{3}\right)$ also splits into $s^{\prime} s^{\prime \prime}$ where $s^{\prime}$ and $s^{\prime \prime}$ are divisors of $q^{\prime}, q^{\prime \prime}$, respectively. Further, we have $r=r^{\prime} r^{\prime \prime}$ and $d=d^{\prime} d^{\prime \prime}$ with $r^{\prime}=q^{\prime} / s^{\prime}$ and $d^{\prime} \mid r^{\prime}$; likewise for $r^{\prime \prime}, d^{\prime \prime}$. Following the proof of Lemma 5 , we now find via the substitution $b=b^{\prime} \frac{r^{\prime \prime}}{d^{\prime \prime}}+b^{\prime \prime} \frac{r^{\prime}}{d^{\prime}}$ that the sum

$$
\sum_{\substack{b=1 \\(b, r / d)=1}}^{r / d}\left|A\left(q^{\prime} q^{\prime \prime}, n-\left(s^{\prime} s^{\prime \prime} d^{\prime} d^{\prime \prime} b\right)^{6}\right)\right|
$$

equals

$$
\sum_{\substack{b^{\prime}=1 \\\left(b^{\prime}, r^{\prime} / d^{\prime}\right)=1}}^{r^{\prime} / d^{\prime}}\left|A\left(q^{\prime}, n-\left(s^{\prime} s^{\prime \prime} d^{\prime} r^{\prime \prime} b^{\prime}\right)^{6}\right)\right| \sum_{\substack{b^{\prime \prime}=1 \\\left(b^{\prime \prime}, r^{\prime \prime} / d^{\prime \prime}\right)=1}}^{r^{\prime \prime} / d^{\prime \prime}}\left|A\left(q^{\prime \prime}, n-\left(s^{\prime} s^{\prime \prime} d^{\prime \prime} r^{\prime} b^{\prime \prime}\right)^{6}\right)\right| .
$$

We may substitute $b^{\prime}$ for $s^{\prime \prime} r^{\prime \prime} b^{\prime}$ and symmetrically for $b^{\prime \prime}$, recalling $\left(s^{\prime \prime} r^{\prime \prime}, r^{\prime}\right)=1$, to rewrite the last product as

$$
\sum_{\substack{b^{\prime}=1 \\\left(b^{\prime}, r^{\prime} / d^{\prime}\right)=1}}^{r^{\prime} / d^{\prime}}\left|A\left(q^{\prime}, n-\left(s^{\prime} d^{\prime} b^{\prime}\right)^{6}\right)\right| \sum_{\substack{b^{\prime \prime}=1 \\\left(b^{\prime \prime}, r^{\prime \prime} / d^{\prime \prime}\right)=1}}^{r^{\prime \prime} / d^{\prime \prime}}\left|A\left(q^{\prime \prime}, n-\left(s^{\prime \prime} d^{\prime \prime} b^{\prime \prime}\right)^{6}\right)\right| .
$$

In this specific sense, the sum over $b$ in 7.9 has a multiplicative property. We break $r$ into $r=u v_{4} w r_{2} r_{3}$ where $r_{j}=v_{j} / s\left(v_{j}\right)^{6}$, and factor the sum over $d$ into independent sums over $d_{j}\left|r_{j}, d^{\prime}\right| u v_{4} w$. Then, by (7.10,

$$
\sum_{\substack{b=1 \\(b, r / d)=1}}^{r / d}\left|A\left(u v_{2} v_{3} v_{4} w, n-s^{6} d^{6} b^{6}\right)\right|=B_{d^{\prime}}\left(u v_{4} w\right) \hat{B}\left(v_{1}, d_{1}\right) \hat{B}\left(v_{2}, d_{2}\right)
$$

where

$$
B_{d}(q)=\sum_{\substack{b=1 \\ b, q / d)=1}}^{q / d}\left|A\left(q, n-(d b)^{6}\right)\right|, \quad \hat{B}\left(v_{j}, d_{j}\right)=\sum_{\substack{b=1 \\\left(b, r_{j} / d_{j}\right)=1}}^{r_{j} / d_{j}}\left|A\left(v_{j}, n-\left(s_{j} d_{j} b\right)^{6}\right)\right| .
$$


By (5.1), we have

$$
\sum_{d \mid q} B_{d}(q)=B(q, n)
$$

and we deduce that $B_{d^{\prime}}\left(u v_{4} w\right) \ll C^{\omega(u)} L^{10}$ because again $B_{d^{\prime}}\left(u v_{4} w\right)$ factorises, the $u$-part is bounded by $C^{\omega(u)}$ in view of Lemma 13 , and by Lemma 11 and (7.1) we see that the $v_{4} w$-part is bounded by $O\left(L^{10}\right)$. We plug this information into 7.9 and factor the sum over $d$ as described in the above discussion. Then

$$
\mathrm{E}_{n}(P) \ll L^{10} \sum_{v_{1} \in \mathcal{V}_{1}}\left|A\left(v_{1}, n\right)\right| \sum_{\substack{u, v_{2} \\\left\{v_{3}, v_{4}, w\right.}} C^{\omega(u)} \sum_{d \mid r} \hat{B}\left(v_{1}, d_{1}\right) \hat{B}\left(v_{2}, d_{2}\right) D_{r / d}^{*}(P /(d s)) .
$$

We now estimate $\hat{B}$ which again factorises according to the prime factorisation of $v_{j}$. Hence, let $p^{t} \| v_{2}$. Then $p^{t}$ is of class 2 , we have $6 \mid t-1$ and $3 \leq t \leq 24$, so that $t=7$ or 13 or 19 . Also, by definition, $s\left(p^{7}\right)=p, s\left(p^{13}\right)=p^{2}$ and $s\left(p^{19}\right)=p^{3}$. Further, with $p^{\nu} \| n$ as usual, we have $\nu \geq 6$ for $t=7$, and $\nu \geq 13$ for $t=13$, and $\nu \geq 19$ for $t=19$. Now, for $d=p^{l}$ with $l=0$ or 1 ,

$$
\hat{B}\left(p^{t}, p^{l}\right)=\sum_{\substack{b=1 \\ p \nmid b}}^{p^{1-l}}\left|A\left(p^{t}, n-p^{t-1+6 l} b^{6}\right)\right| .
$$

This gives $\hat{B}\left(p^{t}, p\right)=\left|A\left(p^{t}, n\right)\right|$ and

$$
\hat{B}\left(p^{t}, 1\right) \leq(p-1) \max _{p \nmid b}\left|A\left(p^{t}, n-p^{t-1} b^{6}\right)\right| .
$$

By Lemma 9, we find that $\hat{B}\left(p^{7}, p\right) \ll 1$ and $\hat{B}\left(p^{7}, 1\right) \ll p$. Similarly,

$$
\begin{array}{ll}
\hat{B}\left(p^{13}, p\right) \ll p\left|A\left(p^{5}, p^{5}\right)\right| \ll p, & \hat{B}\left(p^{13}, 1\right) \ll p \max _{p \nmid b}\left|A\left(p^{13},-p^{12} b^{6}\right)\right| \ll p^{3 / 2}, \\
\hat{B}\left(p^{19}, p\right)=p^{2}\left|A\left(p^{3}, p^{3}\right)\right|=0, & \hat{B}\left(p^{19}, 1\right) \ll p \max _{p \nmid b}\left|A\left(p^{19},-p^{18} b^{6}\right)\right| \ll p^{2} .
\end{array}
$$

In particular, we infer that $\hat{B}\left(v_{2}, d_{2}\right) \ll C^{\omega\left(v_{2}\right)} s\left(v_{2}\right)$, with $C$ sufficiently large. Similarly, for $p^{t}$ of class 3 , we have $p^{6} \| n$ and

$$
\hat{B}\left(p^{8}, p^{l}\right)=\sum_{\substack{b=1 \\ p \nmid b}}^{p^{2-l}}\left|A\left(p^{8}, n-\left(p^{1+l} b\right)^{6}\right)\right| \quad(0 \leq l \leq 2) .
$$

Hence, by Lemma 9 we have $\hat{B}\left(p^{8}, p^{l}\right)=0$ for $l \geq 1$ and

$$
\hat{B}\left(p^{8}, 1\right)=\sum_{\substack{b=1 \\ p \nmid b}}^{p^{2}}\left|A\left(p^{8}, p^{6}\left(n^{\prime}-b^{6}\right)\right)\right|=\sum_{\substack{b=1 \\ b^{6} \equiv n^{\prime} \bmod p}}^{p^{2}}\left|A\left(p^{8}, p^{6}\left(n^{\prime}-b^{6}\right)\right)\right|
$$

in which $n=p^{6} n^{\prime}$ with $p \nmid n^{\prime}$. Hence, there are at most six incongruent solutions of $b^{6} \equiv n^{\prime} \bmod p^{2}$, and at most $6 p$ solutions of $b^{6} \equiv n^{\prime} \bmod p$ with $1 \leq b \leq p^{2}$. By Lemma 9 this shows that $\hat{B}\left(p^{8}, 1\right) \ll p=s\left(p^{8}\right)$. Consequently, we have now shown that

$$
\hat{B}\left(v_{1}, d_{1}\right) \hat{B}\left(v_{2}, d_{2}\right) \ll C^{\omega\left(v_{2} v_{3}\right)} s\left(v_{2} v_{3}\right),
$$


and 7.12 reduces to

$$
\mathrm{E}_{n}(P) \ll L^{10} \sum_{v_{1} \in \mathcal{V}_{1}}\left|A\left(v_{1}, n\right)\right| \sum_{\substack{u, v_{2}, v_{3}, v_{4}, w \\ \text { 7.1. }}} C^{\omega\left(u v_{2} v_{3}\right)} s_{2} s_{3} \sum_{d \mid r} D_{r / d}^{*}\left(P /\left(d s_{1} s_{2} s_{3}\right)\right) .
$$

We apply a dyadic dissection argument according to the size of $s\left(v_{2} v_{3}\right)$. By (7.1), we have $v_{2} v_{3} \leq K$, whence $s_{2} s_{3} \leq\left(v_{2} v_{3}\right)^{1 / 6} \leq K^{1 / 6}$. We cover the interval $[1, K]$ by $O(\log K)$ intervals of the shape $S \leq s\left(v_{2} v_{3}\right) \leq 2 S$ to see that there is an $S \in\left[1, K^{1 / 6}\right]$ with

$$
\mathrm{E}_{n}(P) \ll L^{10}(\log K) S \sum_{v_{1} \in \mathcal{V}_{1}}\left|A\left(v_{1}, n\right)\right| \sum_{\substack{u, v_{2}, v_{3}, v_{4}, w \\ S \leq s\left(v_{2} v_{3}\right) \leq 2 S \\ 7.1}} C^{\omega\left(u v_{2} v_{3}\right)} \sum_{d \mid r} D_{r / d}\left(P /\left(d s_{1} S\right)\right) .
$$

We may now reassemble the complicated summation. We wish to sum over $r$ instead of $u, v_{2}, v_{3}, v_{4}$ and $w$. The number of $v_{2} \in \mathcal{V}_{2}$ producing the same value of $s\left(v_{2}\right)$ is bounded by $24^{\omega\left(v_{2}\right)}$ while $v_{3} \rightarrow s\left(v_{3}\right)$ is a bijection. It follows that at most $24^{\omega\left(v_{2}\right)}$ tuples $u, v_{2}, v_{3}, v_{4}, w$ correspond to the same $r$ while $r$ and $u v_{2} v_{3} v_{4} w$ have the same prime divisors. Hence, $C^{\omega\left(u v_{2} v_{3}\right)} 24^{\omega\left(v_{2}\right)} \leq(24 C)^{\omega(r)}$ and $r \leq Q / v_{1}$. It follows that

$$
\mathrm{E}_{n}(P) \ll L^{11} S \sum_{v_{1} \in \mathcal{V}_{1}}\left|A\left(v_{1}, n\right)\right| \sum_{r \leq Q / v_{1}}(24 C)^{\omega(r)} \sum_{d \mid r} D_{r / d}\left(P /\left(d s_{1} S\right)\right),
$$

with $S$ as before. We write $r=d r^{\prime}$ and exchange the order of summation. Then

$$
\begin{aligned}
\sum_{r \leq Q / v_{1}}(24 C)^{\omega(r)} \sum_{d \mid r} D_{r / d} & \left(\frac{P}{d s_{1} S}\right) \\
\leq & \sum_{d \leq Q / v_{1}}(24 C)^{\omega(d)} \sum_{r^{\prime} \leq Q /\left(d v_{1}\right)}(24 C)^{\omega\left(r^{\prime}\right)} D_{r^{\prime}}\left(\frac{P}{d s_{1} S}\right) .
\end{aligned}
$$

We are ready to apply Lemma 15 . Indeed, $P /\left(d s_{1} S\right) \gg P N^{-\varepsilon} d^{-1} \gg P Q^{-1} N^{-2 \varepsilon} \gg$ $N^{1 / 12}$, and hence we take $Z=P /\left(d s_{1} S\right)$ and $Y=P_{6}^{\eta}$ in Lemma 15 which in view of

$$
\left(\frac{Q}{d v_{1}}\right)^{2} \leq \frac{P}{d s_{1} S} N^{-1 / 80}
$$

are admissible choices. The right hand side of 7.15 is therefore bounded by

$$
\ll \sum_{d \leq Q / v_{1}}(24 C)^{\omega(d)} P S^{-1}\left(d s\left(v_{1}\right)\right)^{-1}(\log N)^{-A} \ll P S^{-1} s\left(v_{1}\right)^{-1}(\log N)^{24 C-A},
$$

with $A$ at our disposal. By (7.14, we arrive at

$$
\mathrm{E}_{n}(P) \ll P L^{11}(\log N)^{24 C-A} \sum_{v_{1} \in \mathcal{V}_{1}} \frac{\left|A\left(v_{1}, n\right)\right|}{s\left(v_{1}\right)} .
$$

Finally,

$$
\sum_{v_{1} \in \mathcal{V}_{1}} \frac{\left|A\left(v_{1}, n\right)\right|}{s\left(v_{1}\right)} \leq \prod_{p^{\nu} \| n}\left(1+\sum_{\substack{t=3 \\ \nu \geq 4}}^{\min (\nu+1,24)} \frac{\left|A\left(p^{t}, n\right)\right|}{s\left(p^{t}\right)}\right) .
$$

For $p^{t}$ of class 1 , we have $s\left(p^{t}\right)=p$ for $3 \leq t \leq 6$, and $A\left(p^{t}, n\right) \ll 1$ by Lemma 9 Similarly, $s\left(p^{t}\right)=p^{2}$ for $8 \leq t \leq 12$ while $A\left(p^{t}, n\right) \ll p$, at least when $\nu$ is so large that these $t$ occur in the sum over $t$. In both cases, we have $A\left(p^{t}, n\right) / s\left(p^{t}\right) \ll p^{-1}$, and this is 
so also in the ranges $14 \leq t \leq 18$ and $20 \leq t \leq 24$ where $s\left(p^{t}\right)=p^{3}$ and $p^{4}$, respectively. This is readily checked via Lemmata 9 and 10 . Hence, the individual factors in (7.17) are of the form $1+O(1 / p)$, showing that (7.17) is bounded by $(\log n)^{\varepsilon}$. In $(7.16$ we take $A$ large and arrive at

$$
\mathrm{E}_{n}(P) \ll P(\log N)^{-1}
$$

8. Singular recovery. This section is a direct continuation of the previous one, and we keep the notation used there. We analyse the sum $\mathrm{M}_{n}(P)$, defined in 7.8 . We will, step by step, reassemble the puzzle and extract an asymptotic formula that features the singular series 1.4 .

The smoothness condition on the divisor $d$ in 7.8 is a nuisance, and we remove this by truncating the $u$-sum appropriately. In fact, by (7.1), the numbers $v_{2}, v_{3}, v_{4}$ and $w$ are all smaller than $P_{6}^{\eta}$, and therefore all their divisors are actually in $\mathcal{A}(Q)$. Let $\mathrm{M}_{n}^{\prime}(P)$ be the portion of the sum $(7.8)$ where $u \leq K$, and let $\mathrm{M}_{n}^{\prime \prime}(P)$ be the complementary part, with $u>K$. Then

$$
\mathrm{M}_{n}(P)=\mathrm{M}_{n}^{\prime}(P)+\mathrm{M}_{n}^{\prime \prime}(P)
$$

and in the sum defining $\mathrm{M}_{n}^{\prime}(P)$ we now have $r<P_{6}^{\eta}$, at least when $N$ is large, so that all $d \mid r$ are appropriately smooth. Hence we can write

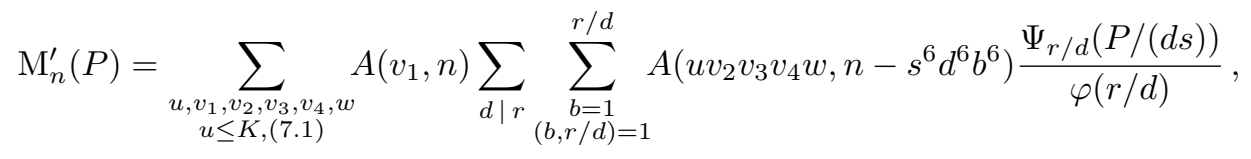

and

$$
\left|\mathrm{M}_{n}^{\prime \prime}(P)\right| \leq \sum_{\substack{u, v_{1}, v_{2}, v_{3}, v_{4}, w \\ u>K, \overline{7.1}}}\left|A\left(v_{1}, n\right)\right| \sum_{d \mid r}\left|\sum_{\substack{b=1 \\(b, r / d)=1}}^{r / d} A\left(u v_{2} v_{3} v_{4} w, n-s^{6} d^{6} b^{6}\right)\right| \frac{\Psi_{r / d}(P /(d s))}{\varphi(r / d)},
$$

The trivial bounds $\Psi_{r / d}(P / d s) \ll P / d s$ and $\varphi(q) \gg q /(\log \log 9 q)^{-1}$ yield

$$
\left|\mathrm{M}_{n}^{\prime \prime}(P)\right| \ll P(\log N)^{\varepsilon} \sum_{\substack{\left.u, v_{1}, v_{2}, v_{3}, v_{4}, w \\ u>K, 7.1\right]}} \frac{\left|A\left(v_{1}, n\right)\right|}{s_{1} s_{2} s_{3} r} \sum_{d \mid r}\left|\sum_{\substack{b=1 \\(b, r / d)=1}}^{r / d} A\left(u v_{2} v_{3} v_{4} w, n-s^{6} d^{6} b^{6}\right)\right| .
$$

Here we consider the sum over $b$. This is similar to the sum considered in the argument leading to 7.10 and $(7.11$, save that $|A|$ is replaced by $A$. Hence, it transpires that the modified analogues of (7.10 and (7.11) are also valid, and in particular, we see that

$$
\sum_{\substack{b=1 \\(b, r / d)=1}}^{r / d} A\left(u v_{2} v_{3} v_{4} w, n-s^{6} d^{6} b^{6}\right)=B_{d^{\prime}}^{*}\left(u v_{4} w\right) \hat{B}^{*}\left(v_{2}, d_{2}\right) \hat{B}^{*}\left(v_{3}, d_{3}\right)
$$

where the notation is the same as in 7.11, and for $d \mid q$ we wrote

$$
B_{d}^{*}(q)=\sum_{\substack{b=1 \\(b, q / d)=1}}^{q / d} A\left(q, n-(d b)^{6}\right), \quad \hat{B}^{*}\left(v_{j}, d_{j}\right)=\sum_{\substack{b=1 \\\left(b, r_{j} / d_{j}\right)=1}}^{r_{j} / d_{j}} A\left(v_{j}, n-\left(s_{j} d_{j} b\right)^{6}\right) .
$$


The triangle inequality gives $\left|B^{*}\left(v_{j}, d_{j}\right)\right| \leq B\left(v_{j}, d_{j}\right)$, and we may apply 7.13 to confirm

$$
\left|\mathrm{M}_{n}^{\prime \prime}(P)\right| \ll P(\log N)^{\varepsilon} \sum_{v_{1} \in \mathcal{V}_{1}} \frac{\left|A\left(v_{1}, n\right)\right|}{s\left(v_{1}\right)} \sum_{\substack{u, v_{2}, v_{3}, v_{4}, w \\ u>K, 7.1}} \frac{C^{\omega\left(v_{2} v_{3}\right)}}{r} \sum_{d \mid r}\left|B_{d^{\prime}}^{*}\left(u v_{4} w\right)\right| .
$$

The sum over $d \mid r$ factors as $d=d^{\prime} d_{2} d_{3}$ with $d^{\prime}\left|u v_{4} w, d_{j}\right| r_{j}$. The sums over $d_{2}$ and $d_{3}$, which are independent of the sum over $d^{\prime}$, contribute $24^{\omega\left(v_{2} v_{3}\right)}$, as we saw in a similar discussion preceding (7.14). Now recalling (7.4) and the analogue of (7.10), this leaves us with

$$
\sum_{d^{\prime} \mid u v_{4} w}\left|B_{d^{\prime}}^{*}\left(u v_{4} w\right)\right|=\sum_{\delta_{1} \mid u}\left|B_{\delta_{1}}^{*}(u)\right| \sum_{\delta_{2} \mid v_{4}}\left|B_{\delta_{2}}^{*}\left(v_{4}\right)\right| \sum_{\delta_{3} \mid w}\left|B_{\delta_{3}}^{*}(w)\right| .
$$

Here the second and the last factor on the right may be bounded trivially, by using the bounds $w \leq L, v_{4} \leq L^{4}$ implied by 7.1 , so that now

$$
\sum_{d^{\prime} \mid u v_{4} w}\left|B_{d^{\prime}}^{*}\left(u v_{4} w\right)\right| \ll L^{10} \sum_{\delta_{1} \mid u}\left|B_{\delta_{1}}^{*}(u)\right| .
$$

Once again by the analogue of 7.10$)$, the sum on the right factors further, according to the decomposition into prime powers. When $t \geq 1$ and $0 \leq l \leq t$, we have

$$
B_{p^{l}}^{*}\left(p^{t}\right)=\sum_{\substack{b=1 \\ p \nmid b}}^{p^{t-l}} A\left(p^{t}, n-\left(p^{l} b\right)^{6}\right) .
$$

If $p \| u$ then 8.6 gives $B_{p}^{*}(p)=A\left(p^{t}, n\right)$ and

$$
B_{1}^{*}(p)=\sum_{b=1}^{p-1} A\left(p, n-b^{6}\right)=\sum_{b=1}^{p} A\left(p, n-b^{6}\right)-A(p, n) .
$$

From 2.1 and 4.6 we see that

$$
\sum_{b=1}^{p} A\left(p, n-b^{6}\right)=p^{-3} \sum_{a=1}^{p-1} S_{2}(p, a) S_{4}(p, a) S_{6}(p, a) S_{8}(p, a) e\left(-\frac{a n}{p}\right),
$$

and we may now imitate the proof of Lemma 7 to show that this expression is bounded by $O\left(p^{-1 / 2}(p, n)^{1 / 2}\right)$. Then, calling also on Lemma 7 itself, we infer that

$$
\sum_{\delta_{1} \mid p}\left|B_{\delta_{1}}^{*}(p)\right| \ll p^{-1 / 2}(p, n)^{1 / 2} .
$$

If $p^{2} \| u$, then by 8.6 ,

$$
B_{p^{2}}^{*}\left(p^{2}\right)=A\left(p^{2}, n\right), \quad B_{p}^{*}\left(p^{2}\right)=(p-1) A\left(p^{2}, n\right),
$$

and with the aid of Lemma 8, we also have

$$
B_{1}^{*}\left(p^{2}\right)=\sum_{\substack{b=1 \\ p \nmid b}}^{p^{2}} c_{p^{2}}\left(n-b^{6}\right)=\sum_{\substack{b=1 \\ b^{6} \equiv n \bmod p \\ p \nmid b}}^{p^{2}} c_{p^{2}}\left(n-b^{6}\right) .
$$


Here we have used the evaluation of the Ramanujan sum. We show that $B_{1}^{*}\left(p^{2}\right)=0$ for all $p \geq 5$ : If there is no solution to $b^{6} \equiv n \bmod p$ with $p \nmid b$, then this is trivial. Otherwise, let $b_{1}, \ldots, b_{h}$ be the solutions of $b^{6} \equiv n \bmod p$ with $1 \leq b \leq p-1$. Then, the solutions of $b \equiv n \bmod p$ with $p \nmid b$ and $1 \leq b \leq p^{2}$ are given by $b_{j}+i p(1 \leq j \leq h, 0 \leq i \leq p-1)$. For each given $j$, exactly one of $b=b_{j}+i p$ satisfies $b^{6} \equiv n \bmod p^{2}$, and therefore, we use again the explicit evaluation of Ramanujan's sum to find that $B_{1}^{*}\left(p^{2}\right)=h\left(\varphi\left(p^{2}\right)-(p-1) p\right)=0$, as anticipated. By Lemma 8, we now have $B_{p^{l}}^{*}\left(p^{2}\right) \ll 1$ for $0 \leq l \leq 2$ where we note that this is trivial for $p \leq 3$. On collecting together, we conclude that whenever $u \in \mathcal{U}$ is factored into $u=u_{1} u_{2}^{2}$ with $u_{1}$ square-free and $\left(u_{1}, u_{2}\right)=1$ then

$$
\sum_{\delta_{1} \mid u} B_{\delta_{1}}^{*}(u) \ll C^{\omega(u)} u_{1}^{-1 / 2}\left(u_{1}, n\right)^{1 / 2} .
$$

By (8.4, 8.5 and (7.4, we now have

$$
\left|\mathrm{M}_{n}^{\prime \prime}(P)\right| \ll P L^{11} \sum_{v_{1} \in \mathcal{V}_{1}} \frac{\left|A\left(v_{1}, n\right)\right|}{s\left(v_{1}\right)} \sum_{\substack{v_{2}, v_{3} \\[7.1}} \frac{(24 C)^{\omega\left(v_{2} v_{3}\right)}}{v_{2} v_{3}} s\left(v_{2} v_{3}\right)^{6} \sum_{u_{1} u_{2}^{2} \geq K} C^{\omega(u)} \frac{\left(u_{1}, n\right)^{1 / 2}}{u_{1}^{3 / 2} u_{2}^{2}}
$$

where we may now sum over $v_{1}$, the pair $v_{2}, v_{3}$, and the numbers $u_{1}, u_{2}$ independently. The $v_{3}$-part is

$$
\ll \sum_{v_{3} \in \mathcal{V}_{3}} \frac{(24 C)^{\omega\left(v_{3}\right)}}{v_{3}} s\left(v_{3}\right)^{6} \ll \prod_{p^{4} \mid n}\left(1+\frac{24 C}{p^{2}}\right) \ll 1,
$$

and for the $v_{2}$-part we recall that $v_{2}$ is composed only of prime powers $p^{7}, p^{13}$, and $p^{19}$, and then find that this contributes

$$
\ll \sum_{v_{2} \in \mathcal{V}_{2}} \frac{(24 C)^{\omega\left(v_{2}\right)}}{v_{2}} s\left(v_{2}\right)^{6} \ll \prod_{p^{4} \mid n}\left(1+24 C\left(\frac{p^{6}}{p^{7}}+\frac{p^{12}}{p^{13}}+\frac{p^{18}}{p^{19}}\right)\right) \ll(\log n)^{\varepsilon} .
$$

We estimated in 7.17 the sum over $v_{1}$ as $O\left((\log N)^{\varepsilon}\right)$. Finally, for the sum over $u_{1}$ and $u_{2}$, we observe that

$$
\begin{aligned}
\sum_{u_{1} u_{2}^{2} \geq K} C^{\omega\left(u_{1} u_{2}\right)} \frac{\left(u_{1}, n\right)^{1 / 2}}{u_{1}^{3 / 2} u_{2}^{2}} \leq \sum_{u_{1}, u_{2}} C^{\omega\left(u_{1} u_{2}\right)}\left(\frac{u_{1} u_{2}^{2}}{K}\right)^{1 / 4} \frac{\left(u_{1}, n\right)^{1 / 2}}{u_{1}^{3 / 2} u_{2}^{2}} \\
\ll K^{-1 / 4} \sum_{u_{1}} C^{\omega\left(u_{1}\right)} \frac{\left(u_{1}, n\right)^{1 / 2}}{u_{1}^{5 / 4}} \ll K^{-1 / 4} \prod_{p \mid n}\left(1+O\left(p^{-3 / 4}\right)\right) \ll K^{-1 / 5} .
\end{aligned}
$$

Using these bounds within 8.4, we deduce the satisfactory bound

$$
\mathrm{M}_{n}^{\prime \prime}(P) \ll P L^{6} K^{-1 / 5} \ll P K^{-1 / 6} .
$$

With the contribution $\mathrm{M}_{n}^{\prime \prime}(P)$ now known to be negligible, we return to the sum $\mathrm{M}_{n}^{\prime}(P)$ presented in 8.2 . The disturbing aspect here is that the term $\Psi_{r / d}$ depends on $d$. This may be removed by appealing to a result of Fouvry and Tenenbaum [6]. Their Théorème 1 implies that

$$
\Psi_{q}(X, Y)=\frac{\varphi(q)}{q} \Psi(X, Y)+O\left(X(\log Y)^{\varepsilon-1}\right)
$$


holds uniformly for $1 \leq \frac{\log X}{\log Y} \ll 1$ and $q \leq X$, which is all we need here. Further, in the same range for $Y$ relative to $X$ we have

$$
\Psi(X, Y)=\varrho\left(\frac{\log X}{\log Y}\right) X+O\left(X(\log X)^{-1}\right)
$$

where $\varrho:(0, \infty) \rightarrow(0,1]$ is the continuous solution of the delay equation $t \varrho^{\prime}(t)=-\varrho(t-1)$ in $t>1$ with $\varrho(t)=1$ for $0<t \leq 1$ (see (1.3) and (1.4) of [4, for example). In our application, we take $q=r / d, Y=P_{6}^{\eta}$ and $X=P /(d s(v))$, and suppose that the conditions of summation in 8.2 are satisfied. Also, recall from 6.2 that $P_{6} / K \leq P \leq P_{6}$. By 7.4 , (7.1) and $u \leq K$ we have $d s \leq u v w \leq K^{2} L^{5}$ and

$$
\frac{\log X}{\log Y}=\frac{\log P}{\log P_{6}^{\eta}}-\frac{\log d s}{\log P_{6}^{\eta}}=\frac{1}{\eta}+O\left((\log N)^{-1 / 2}\right) .
$$

On the one hand, this shows that the asymptotic results for $\Psi_{q}$ and $\Psi$ are applicable, and on the other hand, their use when combined with the mean value theorem and the trivial bound $\left|\varrho^{\prime}(t)\right| \leq 1$ for $t>1$, delivers

$$
\begin{aligned}
\Psi_{r / d}\left(P /(d s), P_{6}^{\eta}\right) & =\frac{\varphi(r / d)}{r / d} \Psi\left(P /(d s), P_{6}^{\eta}\right)+O\left(P(\log N)^{-1 / 2}\right) \\
& =\varrho\left(\frac{1}{\eta}+O\left((\log N)^{-1 / 2}\right)\right) P \frac{\varphi(r / d)}{r s}+O\left(P(\log N)^{-1 / 2}\right) \\
& =\varrho\left(\frac{1}{\eta}\right) P \frac{\varphi(r / d)}{r s}+O\left(P(\log N)^{-1 / 2}\right) .
\end{aligned}
$$

We use this for substitution in 8.2 and find that

$$
\mathrm{M}^{\prime}(P)=\varrho(1 / \eta) P \Sigma_{n}(N)+O\left(P(\log N)^{-1 / 2} \mathrm{H}_{n}(P)\right)
$$

where

$$
\Sigma_{n}(N)=\sum_{v_{1} \in \mathcal{V}_{1}} \frac{A\left(v_{1}, n\right)}{s\left(v_{1}\right)} \sum_{\substack{u, v_{2}, v_{3}, v_{4}, w \\ u \leq K, \text { t.1 }^{7}}} \sum_{d \mid r} \sum_{\substack{b=1 \\(b, r / d)=1}}^{r / d} \frac{A\left(u v_{2} v_{3} v_{4} w, n-s^{6} d^{6} b^{6}\right)}{r s\left(v_{2} v_{3}\right)}
$$

and

$$
\mathrm{H}_{n}(P)=\sum_{v_{1} \in \mathcal{V}_{1}} \frac{\left|A\left(v_{1}, n\right)\right|}{s\left(v_{1}\right)} \sum_{\substack{\left.u, v_{2}, v_{3}, v_{4}, w \\ u \leq K, 7.1\right]}} \sum_{d \mid r}\left|\sum_{\substack{b=1 \\(b, r / d)=1}}^{r / d} \frac{A\left(u v_{2} v_{3} v_{4} w, n-s^{6} d^{6} b^{6}\right)}{r s\left(v_{2} v_{3}\right)}\right| .
$$

The sum in 8.11 is very similar to the right hand side of 8.3$)$, except that now the constraint is $u \leq K$. We therefore follow the transition from (8.3) to 8.8, but do not apply (8.5), as the trivial bounds for the $v_{4}$ and $w$ parts would now be too rough. Instead, we carry these contributions explicitly, from the display preceding (8.5). The $u$-portion changes to a factor no larger than

$$
\left.\ll \sum_{u_{1} u_{2}^{2} \leq K} C^{\omega\left(u_{1} u_{2}\right)} \frac{\left(u_{1}, n\right)^{1 / 2}}{u_{1}^{3 / 2} u_{2}^{2}} \leq \prod_{p \mid n}\left(1+C p^{-1}\right)\right) \ll(\log \log n)^{C},
$$


provided that $C$ is sufficiently large. We then have the temporary estimate

$$
\mathrm{H}_{n}(P) \ll(\log N)^{\varepsilon} \sum_{\substack{v_{4} \in \mathcal{V}_{4} \\ v_{4} \leq L^{4}}} \frac{1}{v_{4}} \sum_{d_{4} \mid v_{4}}\left|B_{d_{4}}^{*}\left(v_{4}\right)\right| \sum_{\substack{w \in \mathcal{W} \\ w \leq L}} \frac{1}{w} \sum_{d \mid w}\left|B_{d}^{*}(w)\right| .
$$

We already observed that the divisor sums over $d$ and $d_{4}$ are multiplicative with respect to $v_{4}$ and $w$. We therefore consider a prime power $p^{t}$ with $p^{t} \| w$. Then $t \geq 3$ and $p \nmid n$. If $d=p^{l}$ with $0 \leq l \leq t$, then 8.6) is applicable. If $6 l \geq t$ we find via Lemma 9 that

$$
B_{p^{l}}^{*}\left(p^{t}\right)=\varphi\left(p^{t-l}\right) A\left(p^{t}, n\right)=0
$$

while for $6 l<t$ we have

$$
B_{p^{l}}^{*}\left(p^{t}\right)=p^{-3 t} \sum_{\substack{a=1 \\ p \nmid a}}^{p^{t}} S_{2}\left(p^{t}, a\right) S_{4}\left(p^{t}, a\right) S_{8}\left(p^{t}, a\right) e\left(-\frac{a n}{p^{t}}\right) \sum_{\substack{b=1 \\ p \nmid b}}^{p^{t-l}} e\left(\frac{a p^{6 l} b^{6}}{p^{t}}\right),
$$

as one confirms from (4.6). Here the sum over $b$ is

$$
\sum_{\substack{b=1 \\ p \nmid b}}^{p^{t-l}} e\left(\frac{a b^{6}}{p^{t-6 l}}\right)=p^{5 l} S^{*}\left(p^{t-6 l}, a\right)
$$

where

$$
S^{*}(q, a)=\sum_{\substack{x=1 \\(x, q)=1}}^{q} e\left(a x^{6} / q\right) .
$$

It follows from Hua [9] that $S^{*}\left(p^{h}, a\right)=0$ whenever $p \nmid a$ and $h \geq 2(p>3)$, and for $h \geq 3$ when $p=3$, and $h \geq 4$ when $p=2$. By 8.14, it follows that $B_{p^{l}}^{*}\left(p^{t}\right)=0$ also holds for $t \geq 6 l+2$ and $p>3$. This leaves the case $t=6 l+1$, which implies $t \equiv 1 \bmod 6$, and here 8.14 reduces to

$$
B_{p^{l}}^{*}\left(p^{6 l+1}\right)=p^{-13 l-3} \sum_{\substack{a=1 \\ p \nmid a}}^{p^{6 l+1}} S_{2}\left(p^{6 l+1}, a\right) S_{4}\left(p^{6 l+1}, a\right) S_{8}\left(p^{6 l+1}, a\right) e\left(-\frac{a n}{p^{6 l+1}}\right) S^{*}(p, a) .
$$

We use (4.11) and the estimate of Hua [9], showing that $S^{*}(p, a) \ll p^{1 / 2}$. Then $B_{p^{l}}^{*}\left(p^{6 l+1}\right) \ll p^{3 l-3 / 8}$ for all $p>3$. The prime $p=3$ is readily handled in the same way, now taking care of the cases $t=6 l+j$ with $1 \leq j \leq 2$, and similarly for $p=2$, with $t=6 l+j, 1 \leq j \leq 2$. In this way we infer that

$$
\sum_{\substack{w \in \mathcal{W} \\ w \leq L}} \frac{1}{w} \sum_{d \mid w}\left|B_{d}^{*}(w)\right| \ll \prod_{p \leq L}\left(1+\sum_{l=0}^{\infty} p^{-1-6 l} p^{3 l-3 / 8}\right) \ll 1 .
$$

The sum over $v_{4}$ in (8.14) can be handled in much the same way. Here $p^{t} \| v_{4}$ implies $p^{4} \mid n$. Recalling the definition of class 4 , we either have $t \geq 25$, or $p^{\nu} \| n$ with $\nu=6,12$ or 18. We begin with $t \geq 25$. For $t>6 l$ we still have 8.14, and so $B_{p^{l}}^{*}\left(p^{t}\right)=0$ for $t \geq 6 l+2$ and $p \geq 5$, as before. For $t=6 l+1$ we can still argue as in the preceding paragraph, providing again the bound $B_{p^{l}}^{*}\left(p^{6 l+1}\right) \ll p^{3 l-3 / 8}$. For $6 l \geq t$ we have 
$B_{p^{l}}^{*}\left(p^{t}\right)=\varphi\left(p^{t-l}\right) A\left(p^{t}, n\right) \ll p^{t-l+[t / 8]} \ll p^{t-2}$ because $t \geq 25$. By an argument similar to the one in 8.15 we now see that the total contribution from primes of class 4 with $t \geq 25$ is a bounded factor.

This leaves prime powers $p^{t}$ with $p^{\nu} \| n, \nu=6,12$ or 18 , and $p^{t} \| q_{9}^{(6)} q_{13}^{(12)} q_{19}^{(18)}$. First consider the case $p^{t} \| q_{9}^{(6)}$. Then $t \geq 9$, and Lemma 9 shows that in this situation 8.13) holds for $6 l \geq t$. Also, via 8.14), we get $B_{p^{l}}^{*}\left(p^{t}\right)=0$ for $t \geq 6 l+2$ except when $p=2$ or 3 . This then leaves the case where $t=6 l+1$, and $t \geq 9$ now enforces $l \geq 2$. We may then work crudely, as in the discussion of the $w$-part above, to see that the primes $p$ with $p^{t} \| q_{9}^{(6)}$ again contribute a bounded factor. The primes dividing $q_{13}^{(12)} q_{19}^{(18)}$ can be dealt with in the same way, showing that an estimate analogous to 8.15 also holds for the $v_{4}$-sum in (8.12), and this establishes the estimate

$$
\mathrm{H}_{n}(P) \ll(\log N)^{\varepsilon} .
$$

We are left with the sum $\Sigma_{n}(N)$, as defined in 8.10 . This is a strangely truncated version of the singular series (1.4). To realise this, we define

$$
T(q, n)=\frac{1}{q^{4}} \sum_{\substack{a=1 \\(a, q)=1}}^{q} S_{2}(q, a) S_{4}(q, a) S_{6}(q, a) S_{8}(q, a) e\left(-\frac{a n}{q}\right)=\frac{1}{q} \sum_{b=1}^{q} A\left(q, n-b^{6}\right) .
$$

The proof of [14, Lemma 2.11] shows that $T(q, n)$ is multiplicative in $q$. We carry out the summation over $d$ in 8.10 and then have

$$
\Sigma_{n}(N)=\sum_{v_{1} \in \mathcal{V}_{1}} \frac{A\left(v_{1}, n\right)}{s\left(v_{1}\right)} \sum_{\substack{\left.u, v_{2}, v_{3}, v_{4}, w \\ u \leq K, 7.1\right]}} \sum_{b=1}^{r} \frac{A\left(u v_{2} v_{3} v_{4} w, n-s^{6} b^{6}\right)}{r s\left(v_{2} v_{3}\right)} .
$$

Here we consider the inner sum over $b$, and recall 77.4 and the definition of a significant divisor. First, according to the remark preceding (7.4), we can use the multiplicative properties of $A$ to sum over $b$ up to $r s^{5}$ instead, obtaining $s^{5}$ copies of the same sum. Hence

$$
\sum_{b=1}^{r} \frac{A\left(u v_{2} v_{3} v_{4} w, n-s^{6} b^{6}\right)}{r s\left(v_{2} v_{3}\right)}=\sum_{b=1}^{r s^{5}} \frac{A\left(u v_{2} v_{3} v_{4} w, n-s^{6} b^{6}\right)}{r s\left(v_{2} v_{3}\right)^{6}},
$$

and then, since $s=s\left(v_{2} v_{3}\right)=s\left(u v_{2} v_{3} v_{4} w\right)$, we find that

$$
\sum_{b=1}^{r} \frac{A\left(u v_{2} v_{3} v_{4} w, n-s^{6} b^{6}\right)}{r s\left(v_{2} v_{3}\right)}=\sum_{y=1}^{r s^{6}} \frac{A\left(u v_{2} v_{3} v_{4} w, n-y^{6}\right)}{r s\left(v_{2} v_{3}\right)^{6}} .
$$

because $A\left(u v_{2} v_{3} v_{4} w, n-y^{6}\right)$ vanishes unless $s \mid y$. By (7.4) and (8.17), the above sum equals $T\left(u v_{2} v_{3} v_{4} w, n\right)$.

We now turn to the sum over $v_{1}$ in 8.18 . By 8.17 the definition of a significant divisor, and 7.3),

$$
T\left(v_{1}, n\right)=\frac{1}{v_{1}} \sum_{\substack{y=1 \\ s\left(v_{1}\right) \mid y}}^{v_{1}} A\left(v_{1}, n-y^{6}\right)=\frac{1}{v_{1}} \sum_{b=1}^{v_{1} / s\left(v_{1}\right)} A\left(v_{1}, n-\left(s\left(v_{1}\right) b\right)^{6}\right)=\frac{A\left(v_{1}, n\right)}{s\left(v_{1}\right)} .
$$


Combining the last two observations, we now conclude from 8.18 that

$$
\Sigma_{n}(N)=\sum_{\substack{u, v_{1}, v_{2}, v_{3}, v_{4}, w \\ u \leq K, 7.1}} T\left(u v_{1} v_{2} v_{3} v_{4} w, n\right) .
$$

Next, we remove the truncations in 8.19. This needs a little care, and we require some upper bounds for $T(q, n)$ which we collect in the following lemma.

Lemma 16. Let $p$ denote a prime, and let $t \in \mathbb{N}$. Then $T\left(p^{t}, n\right) \ll p^{-t / 24}$ holds uniformly in $n$. Further, we have $A(p, n) \ll p^{-3 / 2}(p, n)^{1 / 2}$ and $A\left(p^{2}, n\right) \ll p^{-2}$. If $t \geq 3$ and $p^{4} \nmid n$, then $A\left(p^{t}, n\right) \ll p^{-3 / 2}$ while in the case $p^{4} \mid n$ the weaker bound $A\left(p^{t}, n\right) \ll p^{-1}$ holds.

Proof. By 2.6), we have

$$
T(q, n) \ll q^{-1 / 2} \kappa_{4}(q) \kappa_{6}(q) \kappa_{8}(q),
$$

and if we use this with $q=p^{t}$ for $t \geq 25$, then 2.5 yields the first claim in the lemma. For the estimate of $A(p, n)$ one copies the proof of Lemma 7 Now let $t \geq 2$ and suppose that $6 \nmid t-1$. Then, assuming temporarily that $p>3$, we conclude from (5.2), (5.3) and 2.5 that $p^{-t} S_{6}\left(p^{t}, a\right)=\kappa_{6}\left(p^{t}\right)$ holds whenever $p \nmid a$, and then we infer from (8.17 that $T\left(p^{t}, n\right)=\kappa_{6}\left(p^{t}\right) A\left(p^{t}, n\right)$. The estimates that we claimed for $T\left(p^{t}, n\right)$ are now readily confirmed by referring to Lemmata 6, 8 and 9. This leaves the case where $t \geq 2$ and $t \equiv 1 \bmod 6$. Then by 8.20 and 2.5 , we have $T\left(p^{t}, n\right) \ll p^{-3 / 2}$ for all $t \geq 13$. This leaves the case $t=7$ where 8.20 and 2.5 yield $T\left(p^{7}, n\right) \ll p^{-1}$. This is satisfactory when $p^{4} \mid n$. If $p^{4} \nmid n$ and $p>3$, then $A\left(p^{7}, n\right)=0$. This can be shown by copying the treatment of $A\left(p^{7}, m\right)$ in the proof of Lemma 9. Since $A\left(2^{7}, n\right)$ is bounded, this case is trivial.

With this lemma in hand, the condition $u>K$ in 8.19 is swiftly removed. Indeed, writing again $v=v_{1} v_{2} v_{3} v_{4}$ as on earlier occasions, we have

$$
\sum_{\substack{u, v_{1}, v_{2}, v_{3}, v_{4}, w \\ u>K, 7.1}}|T(u v w, n)| \leq K^{-1 / 4} \sum_{\substack{u, v_{1}, v_{2}, v_{3}, v_{4}, w \\ 7.1}} u^{1 / 4}|T(u, n) T(v, n) T(w, n)| .
$$

Here, we may sum independently over $u \in \mathcal{U}, v \in \mathcal{V}$ and $w \in \mathcal{W}$. By Lemma 16 and multiplicativity, we have

$$
\sum_{v \in \mathcal{V}}|T(v, n)| \leq \prod_{p^{4} \mid n}\left(1+\sum_{t=3}^{\infty}\left|T\left(p^{t}, n\right)\right|\right) \leq \prod_{p^{4} \mid n}(1+O(1 / p)) \ll(\log n)^{\varepsilon}
$$

and

$$
\sum_{w \in \mathcal{W}}|T(w, n)| \leq \prod_{p^{4} \nmid n}\left(1+\sum_{t=3}^{\infty}\left|T\left(p^{t}, n\right)\right|\right) \leq \prod_{p}\left(1+O\left(p^{-3 / 2}\right)\right) \ll 1 .
$$

For the sum over $u$, we first use Lemma 16 to see that $|T(u, n)| \leq C^{\omega(u)}\left(u_{1}, n\right)^{1 / 2} u_{1}^{-3 / 2} u_{2}^{-2}$ where $C$ is sufficiently large and $u=u_{1} u_{2}^{2}$ with $u_{1}$ squarefree and $\left(u_{1}, u_{2}\right)=1$. Then we may carry out the summation over $u$ via 8.7 and find that 8.21 is bounded by $K^{-1 / 5}$. Consequently, we now have

$$
\Sigma_{n}(N)=\sum_{\substack{u, v_{1}, v_{2}, v_{3}, v_{4}, w \\ 7.1}} T\left(u v_{1} v_{2} v_{3} v_{4} w, n\right)+O\left(K^{-1 / 5}\right) .
$$


The conditions summarised in (7.1) involve size constraint on $v_{j}$ and $w$, and these are easily removed by modifications of the preceding argument. By way of example, if one wishes to include terms with $w>L$ (ruled out in (7.1)) in the sum on the right hand side of 8.23 , then one starts from

$$
\sum_{\substack{u, v, w \\ w>L}}|T(u v w, n)| \leq L^{-1 / 96} \sum_{w \in \mathcal{W}} w^{1 / 96}|T(w, n)| \sum_{v \in \mathcal{V}}|T(v, n)| \sum_{u \in \mathcal{U}}|T(u, n)| .
$$

A simple modification of the estimation in 8.22 shows that the sum over $w$ is still bounded while the sum over $v$ is the same as before. For the sum over $u$ we now find

$$
\sum_{u \in \mathcal{U}}|T(u, n)| \leq \sum_{u \in \mathcal{U}} C^{\omega(u)}\left(u_{1}, n\right)^{1 / 2} u_{1}^{-3 / 2} u_{2}^{-2} \ll \prod_{p \mid n}(1+O(1 / p)) \ll(\log n)^{\varepsilon},
$$

and on collecting together, we see that we can remove the condition that $w \leq L$ from 7.1 at the cost of an error not exceeding $O\left((\log N)^{-1}\right)$. Note the similarity of this argument with the derivation of 6.8. All other size conditions in 7.1 can be removed in the same way, and at the same price (recall in particular the method used in $(6.11)$ ), and we then have

$$
\Sigma_{n}(N)=\sum_{q \leq Q} T(q, n)+O\left((\log N)^{-1}\right)
$$

where the sum over $q$ runs through all values that uvw will take. However, uvw runs through all $q$ for which $A\left(q, n-y^{6}\right)$ does not vanish identically as a function of $y \in \mathbb{N}$. But if $A\left(q, n-y^{6}\right)$ vanishes identically, then $T(q, n)=0$ follows from (8.17), and we may include these $q$ in the above expression for $\Sigma_{n}(N)$. Finally, once again using Lemma 16 and converting into an Euler product in the now familiar way, one readily confirms the estimates

$$
\sum_{q} q^{1 / 96}|T(q, n)| \ll \prod_{p^{4} \mid n}\left(1+O\left(p^{-3 / 4}\right)\right) \prod_{p^{4} \nmid n}\left(1+O\left(p^{-33 / 32}\right)\right) \ll n^{\varepsilon}
$$

and

$$
\sum_{q}|T(q, n)| \ll \prod_{p^{4} \mid n}\left(1+O\left(p^{-1}\right)\right) \prod_{p^{4} \nmid n}\left(1+O\left(p^{-33 / 32}\right)\right) \ll(\log \log n)^{C},
$$

provided that $C$ is sufficiently large. Both bounds show that the singular series (1.4) converges absolutely, and the first of these bounds now yields

$$
\Sigma_{n}(N)=\mathfrak{S}(n)+O\left((\log N)^{-1}\right)
$$

while the second delivers the upper bound

$$
\mathfrak{S}(n) \ll(\log \log n)^{C} .
$$

The desired asymptotic formula for $\Upsilon_{n}(P)$ is now available. We successively approximate $\Upsilon_{n}(P)$ via $(6.13),(7.7),(8.1),(8.9)$ and (8.24) while estimating the error terms through (7.18), 8.8) and (8.16). This yields

$$
\Upsilon_{n}(P)=\varrho(1 / \eta) P \mathfrak{S}(n)+O\left(P(\log N)^{\varepsilon-1 / 2}\right)
$$

whenever $P$ and $n$ are in the range 6.2 . The weakest error term in this process comes from 8.9 and 8.16 . Note that this implies the slightly weaker estimate

$$
\Upsilon_{n}(P)=\varrho(1 / \eta) P \mathfrak{S}(n)+O\left(N^{1 / 6}(\log N)^{\varepsilon-1 / 2}\right)
$$


that is valid uniformly for $1 \leq P \leq \frac{1}{2} P_{6}$. Indeed, this certainly follows from 8.26 whenever $P$ is in the range $(6.2)$ while for $P \leq P_{6} \exp (-\sqrt{ } \log N)$ we apply (6.5) and find that in this case $\Upsilon_{n}(P) \ll N^{1 / 6}(\log N)^{-1}$, at least when $N$ is large. Hence, in this case 8.27) also holds.

We now turn the asymptotic relation 8.27 for $\Upsilon_{n}(P)$ into one for $\sigma(n, N)$. The result is as follows.

LEMMA 17. There is a positive number $\mathcal{C}$ such that whenever $\frac{1}{2} N<n \leq N, 8 \nmid n$ and $N$ is sufficiently large, then

$$
\sigma(n, N) \geq \mathcal{C S S}(n) n^{1 / 24}
$$

Proof. We apply partial summation in 4.14 to remove $f_{n}(y)=\left(n-y^{6}\right)^{-1 / 8}$. Note that $f_{n}$ is smooth, and whenever $1 \leq y \leq \frac{1}{2} P_{6}$ and $\frac{1}{2} N<n \leq N$ then $0<f_{n}(y)<\left(\frac{3}{8} N\right)^{-1 / 8}$ and $0<f_{n}^{\prime}(y) \ll N^{-9 / 8} y^{5}$. Hence, by 4.14, 8.25, 8.27) and mundane computations,

$$
\begin{aligned}
\sigma(n, N) & =f_{n}\left(P_{6} / 2\right) \Upsilon_{n}\left(\frac{1}{2} P_{6}\right)-\int_{1}^{\frac{1}{2} P_{6}} f_{n}^{\prime}(t) \Upsilon_{n}(t) \mathrm{d} t \\
& =\varrho\left(\frac{1}{\eta}\right) \mathfrak{S}(n)\left(\frac{1}{2} P_{6} f_{n}\left(\frac{1}{2} P_{6}\right)-\int_{1}^{P_{6} / 2} t f_{n}^{\prime}(t) \mathrm{d} t\right)+O\left(N^{1 / 24}(\log N)^{\varepsilon-1 / 2}\right) \\
& =\varrho\left(\frac{1}{\eta}\right) \mathfrak{S}(n) \int_{1}^{P_{6} / 2} f_{n}(t) \mathrm{d} t+O\left(N^{1 / 24}(\log N)^{\varepsilon-1 / 2}\right) .
\end{aligned}
$$

We substitute $t^{6}=n \tau$ to see that whenever $n \geq 128$ (as we may assume), then $\int_{1}^{P_{6} / 2} f_{n}(t) \mathrm{d} t=\frac{1}{6} n^{1 / 24} \int_{1 / n}^{2^{-6} N / n}(1-\tau)^{-1 / 8} \tau^{-5 / 6} \mathrm{~d} \tau \geq \frac{1}{6} n^{1 / 24} \int_{2^{-7}}^{2^{-6}}(1-\tau)^{-1 / 8} \tau^{-5 / 6} \mathrm{~d} \tau$, and the lemma is immediate.

9. Checkmate. The only missing piece to complete the proof of our main theorem is a lower bound for the singular series.

LEMMA 18. For all $n$ with $8 \nmid n$, one has $\mathfrak{S}(n) \gg(\log \log 9 n)^{-C}$.

Proof. Since $T(q, n)$ is multiplicative in $q$ and the series $\mathfrak{S}$ is already known to be absolutely convergent, we have

$$
\mathfrak{S}(n)=\prod_{p} \mathfrak{s}_{p}(n)
$$

where

$$
\mathfrak{s}_{p}(n)=1+\sum_{t=1}^{\infty} T\left(p^{t}, n\right) .
$$

The proof of Lemma 2.12 of Vaughan [14 provides us with the alternative formula

$$
\mathfrak{s}_{p}(n)=\lim _{h \rightarrow \infty} p^{-3 t} \gamma_{n}\left(p^{h}\right)
$$

where $\gamma_{n}(q)$ is the number of incongruent solutions of $x_{1}^{2}+x_{2}^{4}+x_{3}^{6}+x_{4}^{8} \equiv n \bmod q$. Now, for an odd prime $p$, a straightforward application of the Cauchy-Davenport Theorem, similar to the proof of Lemma 2.15 of Vaughan [14], shows that there is a solution of $x_{1}^{2}+x_{2}^{4}+x_{3}^{6}+x_{4}^{8} \equiv n \bmod p$ with $p \nmid x_{1}$, and then an obvious variant of [14, Lemma 2.13] yields $\gamma_{n}\left(p^{t}\right) \geq p^{3(t-1)}$, so that $\mathfrak{s}_{p}(n) \geq p^{-3}$. For $p=2$ we can argue similarly: since $8 \nmid n$, 
there is a solution of $x_{1}^{2}+x_{2}^{4}+x_{3}^{6}+x_{4}^{8} \equiv n \bmod 8$ with at least one of $x_{1}$ and $x_{3}$ odd, and then the variant of [14, Lemma 2.13] gives $\gamma_{n}\left(2^{t}\right) \geq 2^{3(t-3)}$ for $t \geq 4$ so that $\mathfrak{s}_{2}(n) \geq 2^{-9}$. By Lemma 16 we also have $\mathfrak{s}_{p}=1+O\left(p^{-3 / 2}(p, n)^{1 / 2}\right)$. Hence, for sufficiently large $C$, we get $\left|\mathfrak{s}_{p}-1\right| \leq C p^{-3 / 2}(p, n)^{1 / 2}$. We use this for $p>C^{2}$, and the explicit lower bound for the smaller primes. Then

$$
\mathfrak{S}(n) \gg \prod_{\substack{p \mid n \\ p>C^{2}}}\left(1-\frac{C}{p}\right)
$$

and the lemma follows.

It is time to derive a lower bound for $r(n)$. We assume that $\frac{1}{2} N<n \leq N$, and that

$$
|\varrho(n, N)-\Gamma \sigma(n, N)| \leq N^{1 / 24}(\log N)^{-1 / 2} .
$$

Then, by 3.3 , Lemma 17 and Lemma 18

$$
r(n) \geq \varrho(n, N) \geq \Gamma \mathcal{C S}(n) n^{1 / 24}-N^{1 / 24}(\log N)^{-1 / 2} \geq \frac{1}{2} \Gamma \mathcal{C S}(n) n^{1 / 24}
$$

provided only that $N$ is sufficiently large. However, by Lemma 4 , the number of $n$ with $\frac{1}{2} N<n \leq N$ where 9.1 is false does not exceed $O\left(N^{1-\theta}\right)$. By a dyadic dissection argument, we have now shown the following.

THEOREM. There is a positive number $c$ with the property that $r(n) \geq c n^{1 / 24} \mathfrak{S}(n)$ holds for all but at most $O\left(N^{1-\theta}\right)$ exceptions among the numbers $n \not \equiv 0 \bmod 8$ with $n \leq N$.

Because $\theta>2 / 93$, the version of this result that we stated initially is available through Lemma 18

\section{References}

[1] J. Brüdern, Sums of squares and higher powers. I, J. London Math. Soc. (2) 35 (1987), 233-250.

[2] J. Brüdern, A problem in additive number theory, Math. Proc. Cambridge Philos. Soc. 103 (1988), 27-33.

[3] J. Brüdern, On Waring's problem for cubes, Math. Proc. Cambridge Philos. Soc. 109 (1991), $229-256$.

[4] N. G. de Bruijn, On the number of positive integers $\leq x$ and free of prime factors $>y$, Nederl. Akad. Wetensch. Proc. Ser. A 54 (1951), 50-60.

[5] K. B. Ford, The representation of numbers as sums of unlike powers. II, J. Amer. Math. Soc. 9 (1996), 919-940.

[6] É. Fouvry, G. Tenenbaum, Entiers sans grand facteur premier en progressions arithmetiques, Proc. London Math. Soc. (3) 63 (1991), 449-494.

[7] G. A. Freĭman, Solution of Waring's problem in a new form, Uspehi Mat. Nauk. 4 (1949), no. 1 (29), 193.

[8] G. H. Hardy, E. M. Wright, An Introduction to the Theory of Numbers, 5th edn, Oxford Univ. Press, Oxford 1979.

[9] L.-K. Hua, Some results in the additive prime number theory, Quart. J. Math. Oxford 9 (1938), 68-80. 
[10] K. F. Roth, Proof that almost all positive integers are sums of a square, a positive cube and a fourth power, J. London Math. Soc. 24 (1949), 4-13.

[11] K. F. Roth, A problem in additive number theory, Proc. London Math. Soc. (2) 53 (1951), 381-395.

[12] E. J. Scourfield, A generalization of Waring's problem, J. London Math. Soc. 35 (1960), 98-116.

[13] R. C. Vaughan, A new iterative method in Waring's problem. II, J. London Math. Soc. (2) 39 (1989), 219-230.

[14] R. C. Vaughan, The Hardy-Littlewood Method, 2nd edn, Cambridge Univ. Press, Cambridge 1997.

[15] R. C. Vaughan, T. D. Wooley, Further improvements in Waring's problem, Acta Math. 174 (1995), 147-240.

[16] D. Wolke, Über die mittlere Verteilung der Werte zahlentheoretischer Funktionen auf Restklassen I, Math. Ann. 202 (1973), 1-25. 
